\title{
Horizontal coherence of low-frequency fixed-path sound in a continental shelf region with internal-wave activity
}

\author{
Timothy F. Duda ${ }^{\text {a) }}$ \\ Applied Ocean Physics and Engineering Department, Woods Hole Oceanographic Institution, Woods Hole, \\ Massachusetts 02543 \\ Jon M. Collis \\ Department of Applied Mathematics and Statistics, Colorado School of Mines, Golden, Colorado 80401 \\ Ying-Tsong Lin, Arthur E. Newhall, and James F. Lynch \\ Applied Ocean Physics and Engineering Department, Woods Hole Oceanographic Institution, Woods Hole, \\ Massachusetts 02543 \\ Harry A. DeFerrari \\ Rosenstiel School of Marine and Atmospheric Science, University of Miami, Miami, Florida 33149
}

(Received 14 January 2011; revised 12 July 2011; accepted 14 July 2011)

\begin{abstract}
Sound at 85 to $450 \mathrm{~Hz}$ propagating in approximately 80 -m depth water from fixed sources to a joint horizontal/vertical line array (HLA/VLA) is analyzed. The data are from a continental shelf area east of Delaware Bay (USA) populated with tidally generated long- and short-wavelength internal waves. Sound paths are $19 \mathrm{~km}$ in the along-shore (along internal-wave crest) direction and $30 \mathrm{~km}$ in the cross-shore direction. Spatial statistics of HLA arrivals are computed as functions of beam steering angle and time. These include array gain, horizontally lagged spatial correlation function, and coherent beam power. These quantities vary widely in magnitude, and vary over a broad range of time scales. For example, correlation scale can change rapidly from forty to five wavelengths, and correlation-scale behavior is anisotropic. In addition, the vertical array can be used to predict correlation expected for adiabatic propagation with cylindrical symmetry, forming a benchmark. Observed variations are in concert with internal-wave activity. Temporal variations of three coherence measures, horizontal correlation length, array gain, and ratio of actual correlation length to predicted adiabatic-mode correlation length, are very strong, varying by almost a factor of ten as internal waves pass. (C) 2012 Acoustical Society of America. [DOI: 10.1121/1.3666003]
\end{abstract}

PACS number(s): 43.30.Re, 43.30.Bp [EJS]

Pages: $1782-1797$

\section{INTRODUCTION}

A multi-faceted shallow water observational program known as the Shallow Water 2006 program (also SW06) was undertaken in the late summer of 2006 in waters east of New Jersey and south of Long Island, New York (Tang et al., 2007). This is an area of internal tides (tidal-frequency internal waves) and nonlinear internal-wave packets (Shroyer et al., 2010), with each moving nominally shoreward. Both types of internal waves cause strong and rapid changes in underwater sound propagation behavior. One goal of the program was to measure and explain sound field spatial and temporal variability under these conditions. The intermittent internal-wave induced acoustic fluctuation effects (also known as forward scattering effects) have many signatures. This paper will focus on sound field horizontal structures and sound arrival angles measured with a bottom-resting array, including temporal changes of these quantities. The data used here are receptions of sound at frequencies $85<f<450 \mathrm{~Hz}$ transmitted distances of 20 and $30 \mathrm{~km}$ over

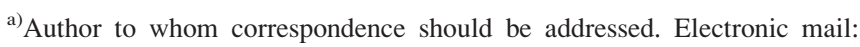
tduda@whoi.edu
}

fixed paths to an L-array, a bottom-resting horizontal line array receiver with a vertical line array at one end.

Figure 1 shows the location of the receiver, one set of sources at a location $30 \mathrm{~km}$ to the northwest (NW sources) and another set of sources at a location $19 \mathrm{~km}$ to the northeast (NE sources). The experimental situation of fixed geometry (source and receiver positions) has less complexity than the moving source/receiver situation and thus can better delineate the observed phenomena. For fixed geometry, time-evolving spatial variation of water-column sound speed by internal waves is the major phenomenon responsible for temporal alterations of sound propagation. (Other phenomena, for instance, surface waves, mesoscale features, and front-linked structures, have somewhat different effects that can be identified and isolated.)

Sound traveling along two types of paths is used. The first path type, from the NW sources, is perpendicular (generally speaking) to internal-wave crests. The second path type, from the NE sources, is approximately aligned with internal-wave crests. The paths from the NE sources are also along-shelf and roughly along-isobath, and are aligned with the shelf edge.

Recent work related to SW06 has demonstrated that passing internal-wave packets can result in a variety of 

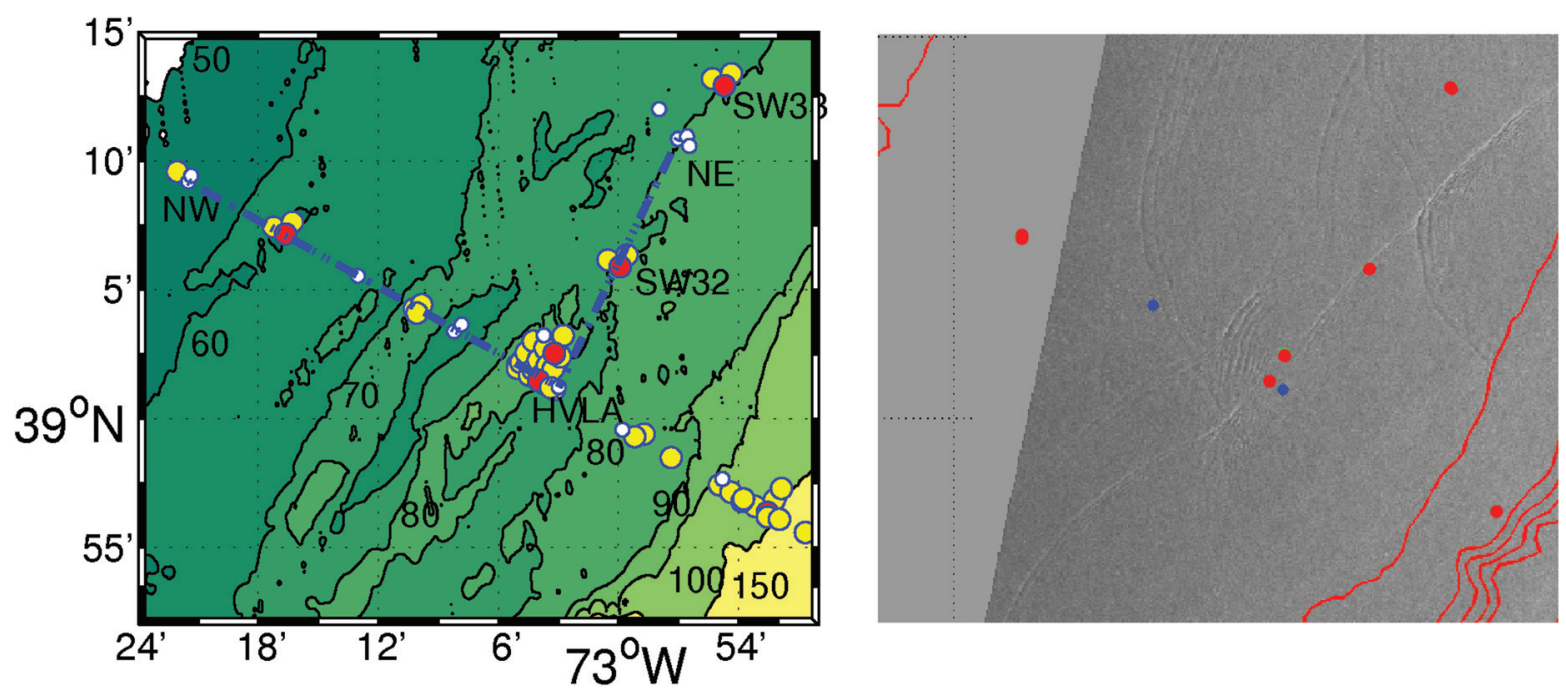

FIG. 1. Left: Chart of the SW06 area east of Cape May, NJ. Contours shown: 50 to $100 \mathrm{~m}$ by $10 \mathrm{~m}$, and $150 \mathrm{~m}$. The white area at the northwest corner is less than $50 \mathrm{~m}$ deep; the dark area is between 50 and $60 \mathrm{~m}$ depth. Lines show the two acoustic paths to the single receiver considered here, with the end points thus showing moored equipment locations. Colored dots correspond to different moorings deployed as part of the experiment. At the "NW" site at the upper left (labeled): WHOI 224-Hz and 400-Hz sources. At the "NE" site at the upper right (labeled): the Miami Sound Machine. At the lower center: the WHOI HVLA receiver. Additional dots show environmental measurement moorings. Red: temperature and current; yellow: temperature; white: temperature plus acoustic or other device. Right: A satellite synthetic aperture radar image is shown. Lines of rough surface corresponding to internal waves are visible. A subset of instrument locations are shown with dots. Red dots show current meter and temperature moorings. Blue dots show wave measurement buoys. Image courtesy of Professor Hans Graber of the University of Miami.

acoustic phenomena that will distort received signals. These include radiation from a truncated internal wave duct (Lin et al., 2009), ducting of acoustic energy down a curved internal-wave trough (Lynch et al., 2010), and horizontal Lloyd's mirror patterns that may result for transmissions incident on internal waves (the Lloyd's mirror direct/ reflected interference pattern stems from a geometrically simple reflector; Lynch et al., 2006). Additionally, daily internal tidal variation of the thermocline depth may impact signal coherence by altering mode excitation by a fixeddepth source.

The appropriate measure of spatial field variation in coherence studies is the correlation function $C(\Delta x)=\langle\psi(x)$ $\left.\psi^{*}(x+\Delta x)\right\rangle$, where $\psi$ is a generalized acoustic field parameter (pressure, complex demodulated pressure, phase-steered pressure, etc.), and where a linear array aligned in the $x$ direction is assumed. Measured correlation functions of demodulated and beam-steered pressure fields and the associated characteristic scales $L_{d}$ of these functions (also named integral scale, or coherence length) are presented here. These yield important information. For example, the coherence length of a received signal governs the usable length of an array. Building on initial results (Collis et al., 2008), this paper presents fixed-path correlation statistics for 22 days of transmissions. Mean $L_{d}$ and variations of $L_{d}$ estimates made over short time windows are each presented, and their behaviors are compared to internal-wave conditions. Also presented are the related quantities array beam power and array signal gain.

The paper is structured as follows. Section II reviews some prior work on this subject. Section III describes the field apparatus. Section IV describes data processing and analysis methods. An appendix contains additional data analysis material. Section V shows horizontal spatial field correlation properties, including correlation length scales and array gain. Section VI contains analysis of the temporal variations of the spatial correlation effects and relates them to internal waves. Section VII summarizes the results.

\section{CORRELATION AND COHERENCE: FUNDAMENTALS AND PRIOR WORK}

\section{A. Fundamentals}

Correlation functions are a standard measure of wave field coherence over both time and space, and are computed and analyzed here. The normalized lagged autocorrelation function $C_{N}$ of the field $\psi$ is defined as

$$
\begin{aligned}
C_{N}\left(x, x^{\prime}, t, t^{\prime}\right) & =\left\langle\psi(x, t) \psi^{*}\left(x^{\prime}, t^{\prime}\right)\right\rangle /\left\langle\psi(x, t) \psi^{*}(x, t)\right\rangle \\
& =C\left(x, x^{\prime}, t, t^{\prime}\right) / C(x, x, t, t)
\end{aligned}
$$

which has unity value when $x=x^{\prime}$ and $t=t^{\prime}$. The angle brackets indicate ensemble average and/or sub-aperture average. The numerator $C\left(x, x^{\prime}, t, t^{\prime}\right)$ is similar to the autocovariance function $C_{0}$, which is the product $C_{0}\left(x, x^{\prime}, t, t^{\prime}\right)$ $=\left\langle(\psi(x, t)-\bar{\psi})\left(\psi\left(x^{\prime}, t^{\prime}\right)-\bar{\psi}\right)^{*}\right\rangle$ involving deviations from the mean of $\psi$ (Emery and Thomson, 2001). For stationary signals, $C$ is independent of $x$ and $t$ so that $C\left(x, x^{\prime}, t, t^{\prime}\right)$ $=C(\Delta x, \Delta t)$, where the displacements $\Delta x=x-x^{\prime}$ and $\Delta t=t-t^{\prime}$ are referred to as lags. The function $C(\Delta x, \Delta t)$ with $\Delta t=0$ is a special case of the more comprehensive mutual coherence function (Frankenthal, 1989).

For signals with a correlation length scale $L_{d}$ longer than a measurement array, subtracting the mean (over the array) from the signals (i.e., computing $C_{0}$ ) will produce a correlation length estimate that is somewhat less than onehalf the array length for all fields, even those with extremely small fluctuations relative to the mean. This can be visualized: After subtracting the mean, the signal will be smooth, 
with positive anomaly at one array end, negative anomaly at the other. Thus $C_{0}$ will be positive at zero lag and trend to negative values at the longest lags. Thus $C_{0}$ is not the quantity of interest here when correlation is high at the longest lag (i.e., for an array that is not significantly longer than $L_{d}$ ), although it is a legitimate and reasonable quantity to compute.

Setting $\psi(x)=A_{0} \exp \left(i \phi_{0}\right)$ and $\psi(x+\Delta x)=A_{1} \exp \left(i \phi_{1}\right)$, one can write $C_{N}(\Delta x)=\left\langle A_{0} A_{1} \exp \left(i\left(\phi_{0}-\phi_{1}\right)\right)\right\rangle /\left\langle A_{0}^{2}\right\rangle^{1 / 2}$ $\left\langle A_{1}^{2}\right\rangle^{1 / 2}=\left\langle A_{0} A_{1}\right\rangle\left\langle\exp \left(i\left(\phi_{0}-\phi_{1}\right)\right)\right\rangle /\left\langle A_{0}^{2}\right\rangle^{1 / 2}\left\langle A_{1}^{2}\right\rangle^{1 / 2}$. The subscript $N$ indicates normalized correlation function with value unity at $\Delta x=0$. With homogenous amplitude distribution (no amplitude trend) this becomes $C_{N}(\Delta x)=\left\langle\exp \left(i\left(\phi_{0}-\phi_{1}\right)\right)\right\rangle$. This is a good assumption for the data analyzed here, with the phase difference factor dominating $C_{N}(\Delta x)$, as is shown in the results section. For Gaussian random phase differences the mutual coherence function expression in the unity gain context is reproduced, $C_{N}(\Delta x)=\exp (-D(\Delta x) / 2)$, where $D$ is the phase structure function defined as $D(\Delta x)=\left\langle\left(\phi_{0}-\phi_{1}\right)^{2}\right\rangle$ (Flatté, 1983).

The function $C_{N}(\Delta x)$ quantifies variations along a wavefront, and provides information regarding coherent signal processing. The relationship between the function $C_{N}(\Delta x)$ and, for example, array processing gain obtained by weighted and/or steered coherent spatial summation $P=\sum_{n=1}^{M} w\left(\mathbf{r}_{\mathbf{n}}\right) \psi\left(\mathbf{r}_{\mathbf{n}}\right)$ (conventional beamformer), where $M$ is the number of array elements at locations $\mathbf{r}_{\mathbf{n}}$ and $w$ are elements of the steering vector, is an interesting one that deserves attention. However, a comprehensive study of this is beyond the scope of this paper. That being said, an expression relating signal-to-noise ratio gain for coherent summation with the correlation function has been derived for a simple exponential form of $C_{N}(\Delta x)$ and linear array geometry (Cox, 1973).

The spatial autocovariance function $C_{0}(\Delta x)$, similar in many instances to the correlation function analyzed here, has some fundamental properties that make it an effective diagnostic tool for acoustic propagation effects, in addition to its diagnostic value for phase-coherent array processing methods which depend on high (or known) spatial correlation properties of a signal (Carey and Moseley, 1991). The autocovariance and the fluctuation spatial-frequency spectrum (the wavenumber $k_{x}$ spectrum) have a well-known Fourier relationship, $C_{0}(\Delta x)=\int_{-\infty}^{\infty} S\left(k_{x}\right) e^{i k_{x} \Delta x} d k_{x}$. It is well known that a narrow $C_{0}(\Delta x)$ falling rapidly away from a maximum at $\Delta x$ equal to zero is indicative of a broad spectrum of spatial wavenumbers, and that a wide $C_{0}(\Delta x)$ falling slowly away from a maximum at $\Delta x$ equal to zero is indicative of a narrow wavenumber spectrum. Both situations arise in the data shown here, with rapid transitions from one to the other.

Figure 2 shows pressure-squared values for two $200-\mathrm{Hz}$ NE pulses, one recorded during a period of weak internalwave activity early on 14 August, and another a few hours later when internal waves were passing over the path. The earlier pulse has strong persistence and has a long apparent coherence length along the HLA. The later pulse shows an interference pattern along the HLA, which carries with it a strong phase pattern (not shown), and has a very short coher-
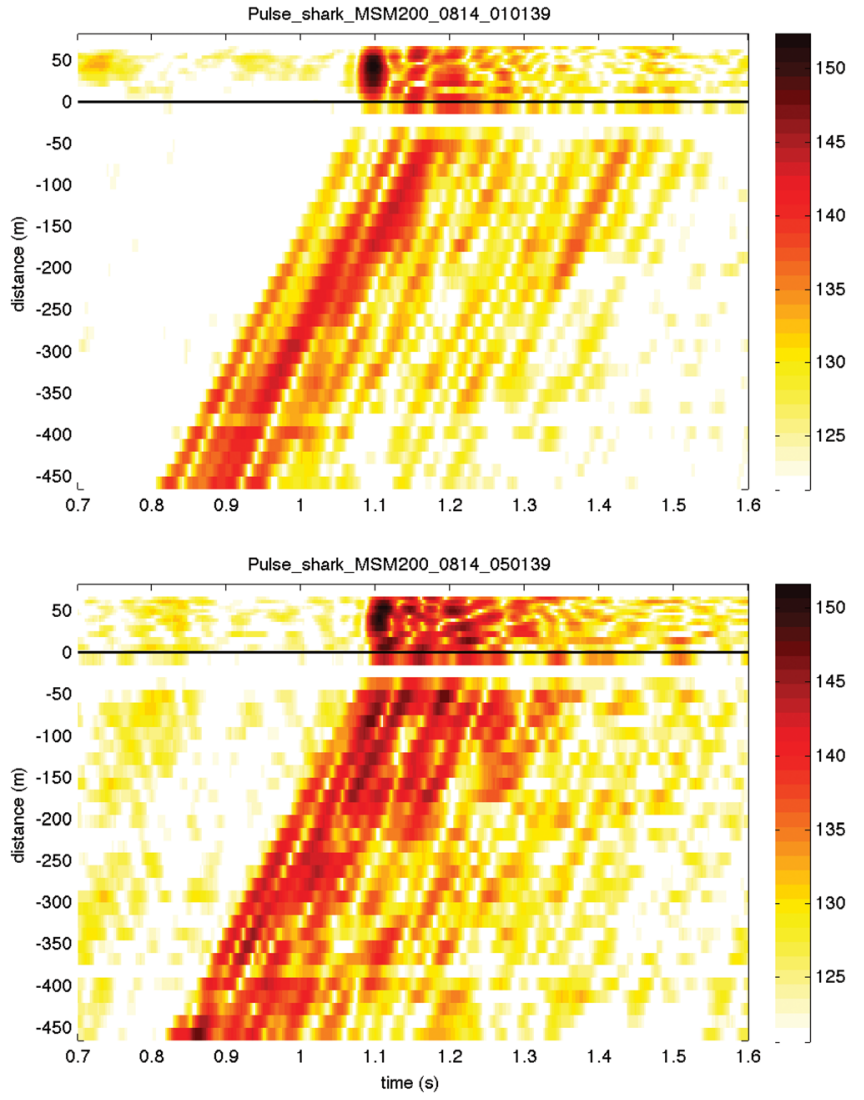

FIG. 2. (Color online) Intensity is shown in dB units for two 200-Hz pulses received at the HVLA from the Miami Sound Machine NE source. The positive distance units depict height above the seafloor along the VLA, the negative units are distance southward from the seafloor data-acquisition unit (anchor of VLA) along the HLA (i.e., the HLA extends northward from the VLA). The upper-frame pulse at 14 Aug 01:01 UTC is during a period of low internal wave activity. The lower-frame pulse at 14 Aug 05:01 UTC shows sound that has passed through a packet of nonlinear internal waves. The 05:01 pulse shows a horizontal interference pattern.

ence length. The earlier pulse, shown in the upper panel of Fig. 2, has a clearly visible dispersed normal mode arrival structure, seen in VLA arrivals measured in the water, at the top of the panel. Modes one, two and three arrive at times $1.10,1.13$, and $1.16 \mathrm{~s}$. The later pulse (lower panel) shows complicated multiple mode arrivals in the VLA data. This could be caused by either mode coupling or strong horizontally varying refraction of adiabatic modes (see Lin et al., 2009 or Lynch et al., 2010). It is not possible to easily determine the cause of multiple mode arrivals by analysis at the receiver alone. At $20 \mathrm{~km}$ range six modes were observed to arrive at $200 \mathrm{~Hz}$, with modes one to six dispersing roughly $0.4 \mathrm{~s}$. This agrees with KRAKEN (Porter, 2011) code modeling using a composite seafloor drawn from a few seafloor models developed in the SW06 program. The model predicts a seventh mode to arrive at $-10 \mathrm{~dB}$ with respect to mode 1 , and an eighth mode at $-23 \mathrm{~dB}$.

Next, note that the spatial interference pattern of a small number of plane waves of the same frequency will scale with the wavelength; in this situation the width (or shape) of $C_{N}(\Delta x, f)$ also scales with acoustic wavelength $\lambda=c / f$, where $c$ is the speed of sound. Such scaling of $C_{N}(\Delta x, f)$ with wavelength has been hypothesized for actual acoustic signals in 
the ocean, but has not been firmly established experimentally. The data presented here have a factor-of- 4 range of $\lambda$ and are suitable for investigating this scaling. The data show that $C_{N}(\Delta x, f)$ is proportional to wavelength at some times, but not at other times. An alternative situation to a few plane waves is sound-beam radiation from a scattering feature, such as a curved internal wave (Lynch et al., 2010), with the beam geometry being a weak function of wavelength. In this situation, $C_{N}(\Delta x, f)$ would be a weak function of $f$, with the width not being proportional to $\lambda$.

\section{B. Prior work}

Many recent studies have examined how packets of nonlinear internal waves, commonly found in the shallow-water environment, perturb sound fields from the simple situation of adiabatic and cylindrically spreading normal modes. Documented effects for two geometries (sound paths perpendicular and parallel to wave crests) are summarized here.

For sound paths perpendicular to crests, strong lateral gradients of sound speed can cause mode coupling. Strong evidence for this has been observed via anomalous signal attenuation (Zhou et al., 1991) and mode multipath arrivals (also called mode arrival spread, Headrick et al., 2000a). The work by Zhou et al. (1991) on coupled-mode propagation through idealized nonlinear internal-wave packets motivated more detailed analysis of mode coupling and mode interference in unitary nonlinear internal waves (Preisig and Duda, 1997). Additional publications covered temporal fluctuations caused by moving nonlinear wave packets (Duda and Preisig, 1999; Rouseff et al., 2002) and fields of waves (Tielbuerger et al., 1997; Headrick et al., 2000b). Follow-on work expanded into analysis of bias effects (net attenuation or amplification) caused by coupled-mode propagation in the presence of two different mode-stripping conditions (Duda, 2004), and into analysis of acoustic field spatial coherence under similar conditions (Duda, 2006; Finette and Oba, 2003; Mignerey and Orr, 2004).

In the other geometry, with sound paths roughly aligned with internal gravity waves, horizontal sound refraction can occur. ("Wave" and "waves" refer to internal gravity waves, henceforth.) The refraction can cause sound convergence within internal-wave ducts or divergence from antiducts (Katsnelson and Pereselkov, 2000; Badiey et al. 2005: Frank et al., 2005), and fluctuating sound radiation from terminating ducts (Lin et al., 2009). Further effects observed as a result of horizontal refraction are sharp intensity fluctuations and frequency-dependent fluctuations (Badiey et al., 2007; Katsnelson et al., 2008; Katsnelson et al., 2009; Badiey et al., 2011), plus rapidly varying modal arrivals, including nulling of modes that would be prominent in the absence of the waves (Lin et al., 2009). Both the across-wave and along-wave situations cause rapid changes in the nature of received acoustic signals. Rapid changes include reduction of horizontal field coherence, creation of phase anomalies consistent with apparent sound arrival angle changes at horizontal arrays, and changes in energy received at specific positions. These three effects are found episodically in the data set examined here.
Previous studies have yielded correlation statistics for the various varieties of the ocean acoustic waveguide (deep sound channel, shallow-water downward refracting, Arctic, etc.). Among them, covariances have been computed for long-range low-frequency propagation (Duda et al., 1992), and horizontal correlation scale estimates have been tabulated (Carey, 1998; Carey et al., 2006). The latter were in the range of tens of wavelengths for shallow water. Spatial correlation of sound passing through internal waves has also been examined with experiments (Orr et al., 2004) and models (Finette and Oba, 2003). These studies show a wide variety of behavior, and are not compatible with concise summary of correlation behavior with respect to a oneparameter description of the internal-wave field (or a few parameters). This paper adds to those works by presenting a large quantity of spatial and temporal correlation measurements collected continuously over a three-plus week period in a shelf area having concurrently measured episodic internal waves. There are two major types of results: Overall average spatial field correlation behavior (Sec. V), and temporal variability of the spatial correlation and its relationship with passing internal waves (Sec. VI).

As for temporal fluctuations, shelf acoustic propagation has been demonstrated through experiment to be highly variable in time. Specific experiment locations are the MidAtlantic Bight shelf (Headrick et al., 2000a, Fredricks et al., 2005) and the western side of the northern South China Sea (Duda et al., 2004; Chiu et al., 2004; Mignerey and Orr, 2004; Duda et al., 2011). Much of the variability can be attributed to the presence of moving internal gravity waves and wave packets.

In addition to these studies of internal-wave related coherence of sound at frequencies below $1500 \mathrm{~Hz}$, other studies of underwater sound spatial coherence are available. Many of these have insightful discussions of effects causing limited spatial correlation (Berkson, 1980; Dowling and Jackson, 1993; Meredith and Nagle, 1999; Dahl, 2004).

\section{DESCRIPTION OF THE EXPERIMENT}

The SW06 field program was a large multinational study of acoustic propagation and nonlinear internal wave physics (Tang et al., 2007). Lasting for two months, the experiment employed seven ships, 62 moorings, aircraft overflights, satellite imagery, autonomous underwater vehicles, real-time data communications, and numerical modeling. Moored acoustic systems were in place from late July until early September, 2006, along with approximately forty moorings dedicated to environmental measurements. Data analyzed here were collected between 3 August and 25 August. Figure 1 shows the experimental area. The lines in the figure show the paths between the northwest (NW) and northeast (NE) source groups and a large moored receiver system emplaced by Woods Hole Oceanographic Institution (WHOI). The acoustic data used here are from these instruments. The receiver consisted of a 32-element horizontal line array (HLA) with a 16-channel vertical line array (VLA) tied to one end. This is referred to as the HVLA. The depth at the HVLA site was $79 \mathrm{~m}$. 
The basic mooring suite was deployed in a " $T$ " shaped geometry (Fig. 1). One leg of the T, containing the NE to HVLA path, had an along-shelf alignment following (approximately) the $80 \mathrm{~m}$ isobath, at bearing $30^{\circ}$ (true). The other leg was aligned across-shelf from depths of 58 to $500 \mathrm{~m}$ at bearing $300^{\circ}$. The NW to HVLA path was along a portion of this leg. The NE and NW sites were 19.2 and $30.2 \mathrm{~km}$ from the HVLA, respectively. The right side of Fig. 1 shows one lowresolution ERS-2 satellite synthetic aperture radar image of internal waves on 8 August 2006. The image shows approximate alignment of internal-wave crests with the NE-HVLA path, a typical situation for this area (Lynch et al., 2010)

The moored sources at the NW and NE sites transmitted lengthy phase-encoded $\mathrm{M}$-sequence signals that were matched-filter processed to form short synthetic pulses, with the processed pulses repeating at the sequence-length interval (between 2.4 and $5.1 \mathrm{~s}$ for the four sources). The NElocated "Miami Sound Machine" (MSM) source was moored approximately $12 \mathrm{~m}$ above the seafloor in 79-m deep water. The MSM signals had center frequencies of 100, 200, 800, and $1600 \mathrm{~Hz}$ and bandwidths of about one-quarter the center frequencies. Signals at 100 and $200 \mathrm{~Hz}$ are examined here. At NW, two coded-signal sources at 224 and $400 \mathrm{~Hz}$, with $16-\mathrm{Hz}$ and $100-\mathrm{Hz}$ bandwidths, respectively, were moored $500 \mathrm{~m}$ apart. The NW water depth was $58 \mathrm{~m}$ and each sound source was located $9 \mathrm{~m}$ above the bottom. Further details are available in a report (Newhall et al., 2007).

Environmental conditions were recorded at the HVLA receiver, at the source locations, and between the HVLA and the sources. Vertical velocity and temperature measurements at these locations (covering most of the water column) give precise information about background conditions and internalwave events, including wave structures in the vertical, speeds, and bearings. The physical oceanography measurement moorings were arranged to provide good along- and crossshelf coverage of the shelf-break front, the local eddy field, the internal tide, and the nonlinear internal wave field.

Essential properties of the 48-channel HVLA system are described here. The data-acquisition unit (Shark) was on the seafloor. Above the Shark was a 16-element vertical array of variable spacing, with topmost element at $13.5 \mathrm{~m}$ depth, so that the Shark served as a deadweight anchor for the VLA. The 32-element HLA was $465 \mathrm{~m}$ in length (15-m element spacing) and extended northward from the Shark, with the southernmost element $7 \mathrm{~m}$ north of the Shark. For the NE and NW sources, nominal angles of incidence $\theta_{i}$ at the HLA center are $\theta_{1}=\theta_{2}=26.0^{\circ}$ for the 100 and $200 \mathrm{~Hz}$ signals, $\theta_{3}=-61.56^{\circ}$ for the $224 \mathrm{~Hz}$ signal, and $\theta_{4}=-60.55^{\circ}$ for the $400 \mathrm{~Hz}$ signal. (Broadside from the east is $90^{\circ}$. The subscript indicates $100 \mathrm{~Hz}$ source, $200 \mathrm{~Hz}$ source, etc.) The HLA was not exactly straight in the north-south direction. In fact, the shape changed between 10:35 and 11:00 UTC on the 19th of August during a nonlinear internal wave event. The array shape anomalies before and after the event, projected in the NE source direction, are shown in Fig. 12 of Appendix B. The actual anomalies transverse to the array direction are greater, being that the values in the figure are those divided by $\sin \left(26^{\circ}\right)$. Prior to the event the array bowed eastward from the Shark (the Shark could not move) for half of its length with a maximum anomaly of $4 \mathrm{~m}$ at $225 \mathrm{~m}$ north of the shark, then bowed back to the west to have an eastward anomaly of $1 \mathrm{~m}$ at the northern end. The event moved the array from east of the Shark meridian to west of it, settling into a shape with maximum anomaly of $2.5 \mathrm{~m}$ westward at the northern end. These HLA shapes were determined in the procedure used to calculate the compensating steering vector $V(x, \theta)$, where $\theta$ is the steering angle. The procedure is described in the next section.

Although the along-shelf propagation path roughly tracks the $80 \mathrm{~m}$ isobath, the terrain has sand ridges and scours that alter the depth along the path. The cross-shelf path begins at $58 \mathrm{~m}$ depth and passes over a bump and a scour before finishing at the bottom-resting HLA $(80 \mathrm{~m}$ depth). The depth excursions are gradual, consistent with adiabatic mode propagation, and inconsequential to the decorrelation effects found here.

\section{DATA ANALYSIS METHODS}

\section{A. Beam-steered correlation functions}

The horizontal spacing of the HLA elements will impart phase shifts of the source signals along the array, and planewave beam steering is used here to counteract the reducing effect this will have on coherence (Johnson and Dudgeon, 1993). With zero noise and plane waves, the correlation function will have the maximum value of one for all lags after beam steering. Finite noise will reduce this, as will signal departure from plane waves. The signal-to noise ratio is high for the coded sources, so the primary contributor to reduced coherence is departure from plane wave geometry. The sources are not truly far-field, and point-source beam steering would provide a measure of maximum array gain for our signals. The point-source phase correction method is combined here with the bent-array phase correction. A correction vector $V\left(x, \theta_{1}\right)=\exp \left[i \phi\left(x, \theta_{1}\right)\right]$ for HLA location $x$ and steering angle $\theta_{1}$ is devised from the data set itself. For plane waves at a straight array the phase would be $\phi=2 \pi x \cos \theta_{1} / \lambda$. The vector $V\left(x, \theta_{1}\right)$ used here compensates for effects of both array bending (departure from a straight line) and wavefront curvature for the NE sources.

The vector $V\left(x, \theta_{1}\right)$ is found by analyzing demodulated phases within plane-wave beam-steered, resolved dispersed mode-one transmissions at $100 \mathrm{~Hz}$ from the MSM. A steering vector $V\left(x, \theta_{1}\right)$ that is complex conjugate to the long-term median mode-one phases $\Phi_{1}(x)$ along the array is generated. The NE $100-\mathrm{Hz}$ source curved wavefront has a maximum anomaly at the center of about $0.2 \mathrm{~m}$ (zero anomaly from a plane-wave front at the north and south ends, 0.17 radians at $200 \mathrm{~Hz}$, half that at $100 \mathrm{~Hz}$ ). The array shapes mentioned in the prior section are then computed with the good assumption that the array shifts east-west and the Kevlar strength member does not stretch. The dislocations are given by $\Delta y(x)=\lambda_{100} \Phi_{1}(x) / 2 \pi \sin \theta_{1}=r_{100}(x) / \sin \theta_{1}$, where $r_{100}(x)$, plotted in Fig. 12, is equivalent wavefront deformation. The NW correction $V\left(x, \theta_{3}\right)$ is computed from $\Delta y(x)$. The phase adjustments in $V$ are a factor of ten greater than predicted curvature adjustments. 
A hybrid method composed of time-delay processing and steering-vector multiplication is used for the digital steering processing. The processed signal at position indicated by index $n$ along the array is

$$
\psi_{A}\left(x_{n}, \theta, t\right)=P\left(x_{n}, t+m(n, \theta) \delta t\right) V\left(x_{n}, \theta\right),
$$

where $P$ is the complex demodulated pressure after matchedfilter processing. The time delay for each element is the integer $m(n, \theta)$ multiplied by the sampling interval $\delta t$ of $P$. The best time shifts for an oversampled signal would be $T_{n}=x_{n} \cos \theta / c ; m(n, \theta)$ is the closest integer to $T_{n} / \delta t$. The time delay preserves amplitude variations along the array and has no effect on phase. The steering vector $V$ affects only phase. Signal correlation along the array is significantly higher for $\psi_{A}\left(x_{n}, \theta, t\right)=P\left(x_{n}, t+m(n, \theta) \delta t\right) V\left(x_{n}, \theta\right)$ than for $\psi_{A}\left(x_{n}, \theta, t\right)=P\left(x_{n}, t+m(n, \theta) \delta t\right)$, affirming the effective phase compensation. Use of the function $V$ for NW signal analysis imparts a small error because the (weak) NE curvature is erroneously introduced, and the (weak) NW curvature is not accounted for. Removing the NE curvature analytically is expected to have little effect on the results because the curvature phase values are small compared to the bending values and to observed fluctuating phase values along the array. The reason for choosing this plane-wave-based method, with an ad-hoc curvature correction for NE sources, is that the results will be meaningful for describing expected signal variations for generic shallow-water sources whose ranges are not precisely known and therefore could not be analyzed with point-source beam steering.

The primary quantity analyzed in this paper is a statistic that is similar to $C_{N}(\Delta x)$. It is the normalized correlation function for time integrals

$$
\begin{aligned}
& R_{N}(\Delta x)=\left\langle\frac{\int_{t_{1}}^{t_{2}} \psi_{A}(x, t) \psi_{A}^{*}(x+\Delta x, t) d t}{\left(\int_{t_{1}}^{t_{2}}\left|\psi_{A}(x, t)\right|^{2} d t \int_{t_{1}}^{t_{2}}\left|\psi_{A}(x+\Delta x, t)\right|^{2} d t\right)^{1 / 2}}\right\rangle \\
& \cong \frac{\left\langle\int_{t_{1}}^{t_{2}} \psi_{A}(x, t) \psi_{A}^{*}(x+\Delta x, t) d t\right\rangle}{\left\langle\int_{t_{1}}^{t_{2}}\left|\psi_{A}(x, t)\right|^{2} d t\right\rangle}=\frac{R(\Delta x)}{R(0)} .
\end{aligned}
$$

The approximation is true for normally distributed stationary signals and is not utilized here; it shows the relationship between various quantities of interest. The normalization before averaging ensures that the correlation behaviors, versus lag, of weaker and stronger signals are given equal weight in the averaging procedures used in forming $R_{N}(\Delta x)$. The relationship of this broadband correlation function to other measures is examined in Appendix A. In this study the integral is replaced by discrete summation at the reduced sampling rate of the matched-filter processed pulses, which is usually at twice the center frequency (the center frequency may also be considered as the carrier frequency of the phase modulated signal). The coherence length $L_{d}$ along the array is defined here as the $\Delta x$ point where $R_{N}(\Delta x)$ drops below $1 / e \sim 0.37$. The value of analyzing the compressed pulses is evident when the effects of noise are considered. Correlation values estimated from field data are actually

$$
\begin{aligned}
R(\Delta x)= & \left\langle\int _ { t _ { 1 } } ^ { t _ { 2 } } [ \psi _ { S } ( x , t ) + v ( x , t ) ] \left[\psi_{S}^{*}(x+\Delta x, t)\right.\right. \\
& +v(x+\Delta x, t)] d t\rangle
\end{aligned}
$$

where $\psi_{S}$ is signal from a known source and $v$ is noise, i.e., $\psi_{A}=\psi_{S}+v$. This breaks into four integrals. Any finite crosscorrelation of the two noise series or the signal and noise series (possible for dominant noise arriving at the same angle as the signal) introduces error into $R$ estimation. This error is reduced by using $\psi$ that are enhanced by matched-filter processing gains of $18 \mathrm{~dB}$ (100 and $225 \mathrm{~Hz}$, using 63 digits), $21 \mathrm{~dB}(200 \mathrm{~Hz}$, 127 digits), and $27 \mathrm{~dB}$ (400 Hz, 511 digits). Estimates for $R$ were computed for the 100,200, 224, and $400 \mathrm{~Hz}$ sources using time subintervals of the received signals $\left(t_{2}-t_{1}\right)$ of $0.4,0.2$, 0.31 , and $0.12 \mathrm{~s}$, with $t_{1}$ at the leading edge of the pulse. These time intervals include the portion of the received signal with significant amplitude above noise levels. Because the array had 32 elements, there are many degrees of freedom in $R$ estimates for short lags, with the number of degrees of freedom declining with lag. Individual-pulse $R$ functions are reliable and provide usable $L_{d}$ estimates. Estimates of $R$ made over a few hours contain even more degrees of freedom [six (single-sequence) pulses per hour are processed].

Figure 3 shows $R_{N}(\Delta y, \theta)$ for $200-\mathrm{Hz}$ data computed using a 2 -h averaging interval, where $\theta$ is HLA steering angle. A line on the plot shows the $\exp (-1)$ contour of $R_{N}(\Delta y, \theta)$, i.e., the line shows $L_{d}(\theta)$. The grating lobes can be seen at low and high angles.

With the processing methods explained, the veracity of the assumption $C_{N}(\Delta x)=\left\langle\exp \left(i\left(\phi_{0}-\phi_{1}\right)\right)\right\rangle$ can be examined. This assumption is not depended on here, but often an equivalent assumption is made and phase alone is used in theoretical and experimental array-processing work. This relationship is close to being true for these data, with the phase difference factor dominating $C_{N}(\Delta x)$. Figure 3 illustrates this by examining $\left\langle A_{0} A_{1}\right\rangle /\left\langle A_{0}^{2}\right\rangle$ versus $\left\langle A_{0} A_{1} \exp \left(i\left(\phi_{0}-\phi_{1}\right)\right)\right\rangle /\left\langle A_{0}^{2}\right\rangle$, with the former having high correlation at all lags.

There are a few sources of error for $C_{N}(\Delta x)$ $=\left\langle\exp \left(i\left(\phi_{0}-\phi_{1}\right)\right)\right\rangle$. Introducing phase differences arising from true signal, wavefront curvature, array bending, and noise, one has

$$
\begin{aligned}
C_{N}(\Delta x)= & \langle\exp (i \Delta \phi)\rangle=\left\langle\operatorname { e x p } \left(i \Delta \phi _ { T } \left( 1+\Delta \phi_{C} / \Delta \phi_{T}\right.\right.\right. \\
& \left.\left.\left.+\Delta \phi_{B} / \Delta \phi_{T}+\Delta \phi_{v} / \Delta \phi_{T}\right)\right)\right\rangle
\end{aligned}
$$

These data have high signal to noise ratio, so the last term is small. The array bending term is accounted for in our processing and is reduced to a small fraction of one. The curvature term is also small, leaving the true phase difference $\Delta \phi_{T}$.

One comment on stationarity must be included here: The time frame considered to be stationary in the derivation in Appendix A is that of the compressed pulse, which is on 
a) Real $R_{N}(\Delta x, \theta) ; 14.0$ to $14.08(\mathrm{hr} 0-2)$

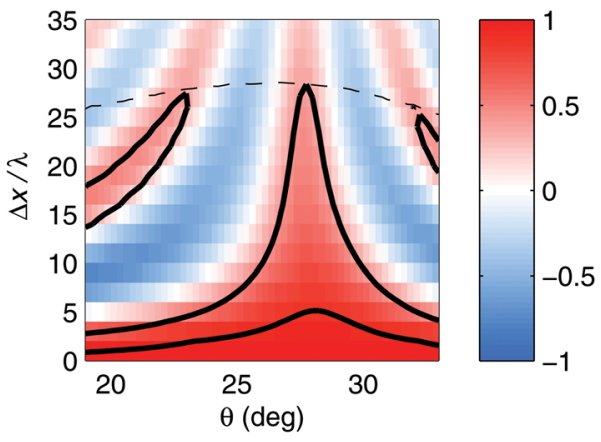

c) Real $R_{N}(\Delta x, \theta) ; 14.25$ to $14.33(\mathrm{hr} 6-8)$

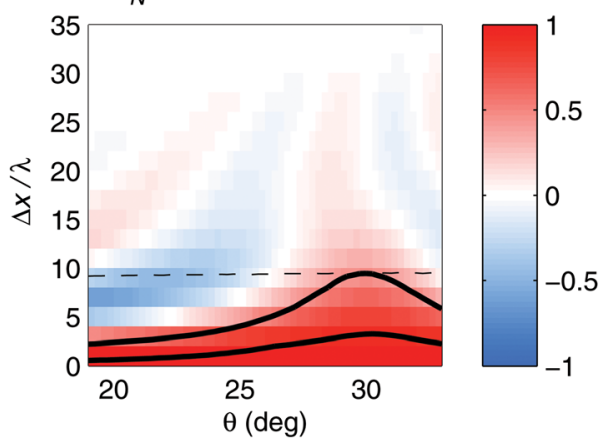

b) $R_{I N}(\Delta x, \theta) ; 14.0$ to $14.08(\mathrm{hr} 0-2)$

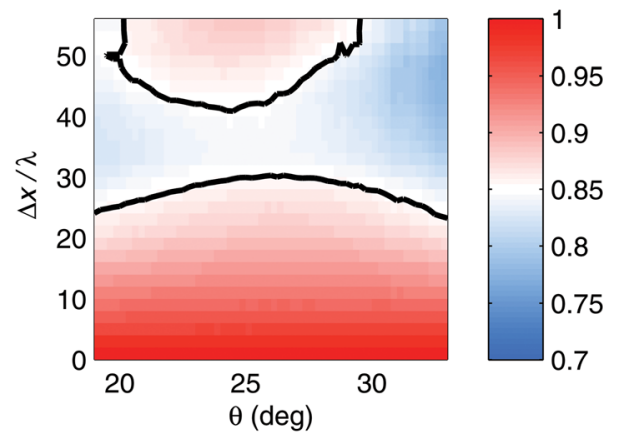

d) $R_{I N}(\Delta x, \theta) ; 14.25$ to $14.33(\mathrm{hr} 6-8)$

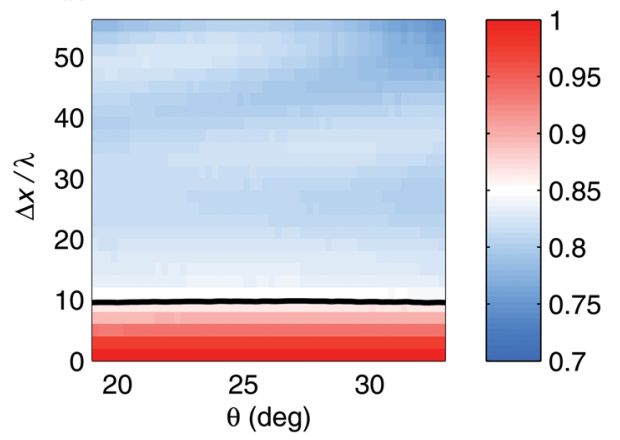

FIG. 3. (Color online) (a) The real part of $R_{N}=\langle\psi * \psi\rangle$ as a function of steering angle is shown for the $200-\mathrm{Hz}$ source for the interval 00:00-02:00 of August 14. The contour lines are at 0.85 and $1 / e$. The dashed line is the $1 / e$ contour of $\left|R_{N}\right|$. The plot shows a maximum coherence scale of $L_{d}=28$ wavelengths. (b) The real quantity $R_{I N}=\langle|\psi * \|| \psi \mid\rangle=\left\langle A_{0} A_{1}\right\rangle$ is shown, with one contour at $R_{I N}=0.85$ having peak value of 30 , compared with 5 in (a). This shows that $|\psi|$, which is often plotted for broadband array arrivals, has a longer coherence scale than $\psi$. (c) The same thing is shown as in (a), but for a later time period (06:00-08:00 on August 14) with shorter coherence scale than for the other time period. (d) The quantity shown in (b) is again shown, for the 06:00-08:00 period. the order of seconds. Changes of $R$ over periods of days or hours are examined later in the paper, suggesting nonstationarity. These changes are observed after temporal averaging as in the above equation and then ensemble averaging over intervals of minutes to a day, assumed to be quasistationary.

\section{B. Horizontal array gain and beam power}

The horizontal array gain is computed for the four sources and compared to theoretical limits. Gain of the array is defined as the signal power $(S)$ to noise power $(\tilde{N})$ ratio for the coherent sum divided by the same ratio for a single element,

$$
G(M)=\left\langle\frac{S(M)^{2}}{\tilde{N}(M)^{2}}\right\rangle\left\langle\frac{S(1)^{2}}{\tilde{N}(1)^{2}}\right\rangle^{-1}
$$

where $M=2^{n}, n=0$ to 5 , is the number of array elements. The summed $S(M)$ (signal) and $\tilde{N}(M)$ (noise) values are computed with full array $(M=32)$ or sub-array processing (i.e., $M \leq 16$, also called sub-aperture processing) and have the highest statistical reliability at the short lags, which have the most plentiful sub-apertures. Noise levels are found using matched-filter output at times with no signal (in gaps between matched-filter arrivals).

Beam power is computed from the array sum of received signal after beam steering. It is defined here as the squared sum of the steered pressure fields along elements of the horizontal array

$$
B P=\left|\sum_{n=1}^{M} \psi_{A n}\right|^{2}
$$

For the full HLA these have 29 to 32 elements, as three hydrophones were intermittent. The quantity $B P$ is not emphasized in this paper, appearing in one figure. The causes of beam power variation may differ from the causes of coherence loss, and the relationship between these two remains to be examined in detail.

\section{Synthetic pulse cross-correlation functions}

A joint HLA/VLA processing method for separating two distinct decorrelation processes for oblique incidence HLA receptions is used here, as in Collis et al. (2008). The two processes are azimuthally dependent propagation and range-dependent modal interference of an azimuthally uniform field. Decorrelation from the latter process is estimated and then compared with total decorrelation to estimate the former. Under the assumption of cylindrically symmetric propagation, a synthetic acoustic demodulated pressure field $p_{S}$ along the HLA can be computed using modal decomposition of pulse signals measured with the nearby VLA. The decomposition is written $p_{V L A}\left(x_{0}, y_{0}, z, t\right)$ $=\sum_{n=1}^{T} A_{n}(t) \phi_{n}(z, t)$, where $A_{n}$ are the complex mode amplitudes, $\phi_{n}$ are the mode shapes, and the total number of modes is $T$. With azimuthal uniformity and no mode coupling, the modal signals determine pressure fields anywhere in the waveguide, including at the HLA positions. The projected distances $\Delta \eta$ toward the source of any location, relative to the VLA position, and the modal wavenumbers $k_{n}(t)$ are then used to compute the synthetic field

$$
p_{S}(x, y, z, t)=\sum_{n=1}^{N} A_{n}(t) \phi_{n}(z, t) \exp \left(i k_{n}(t) \Delta \eta(x, y)\right)
$$



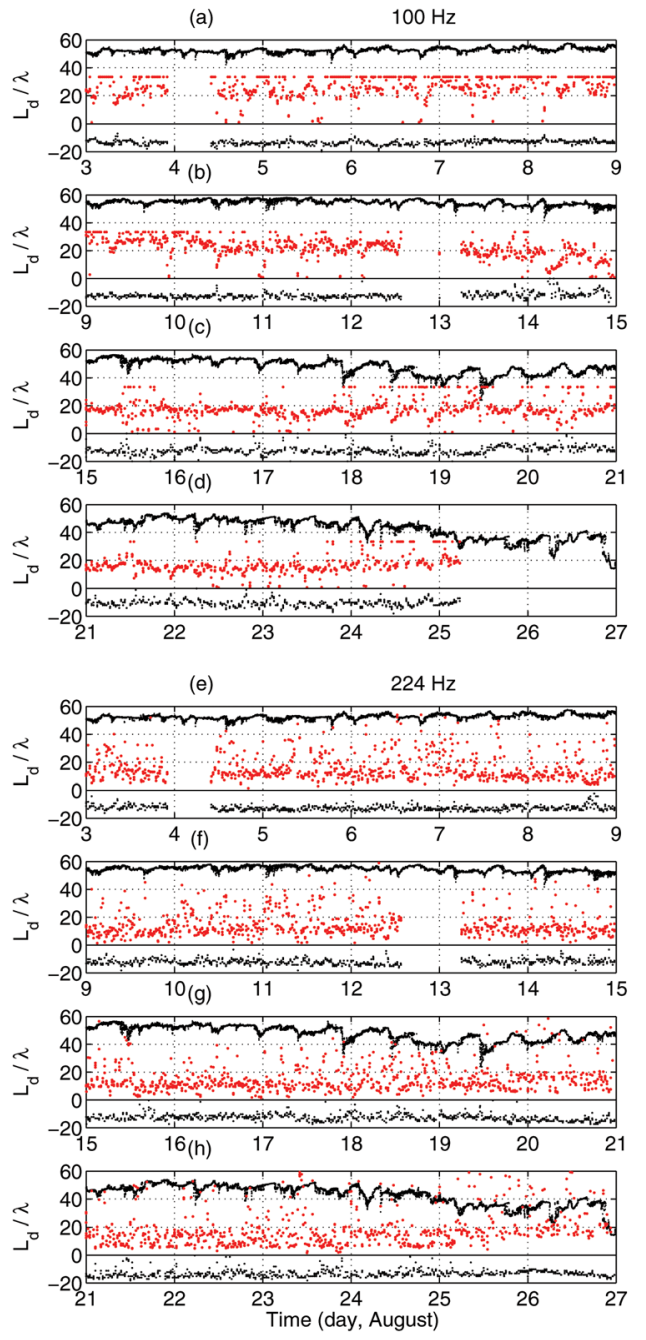
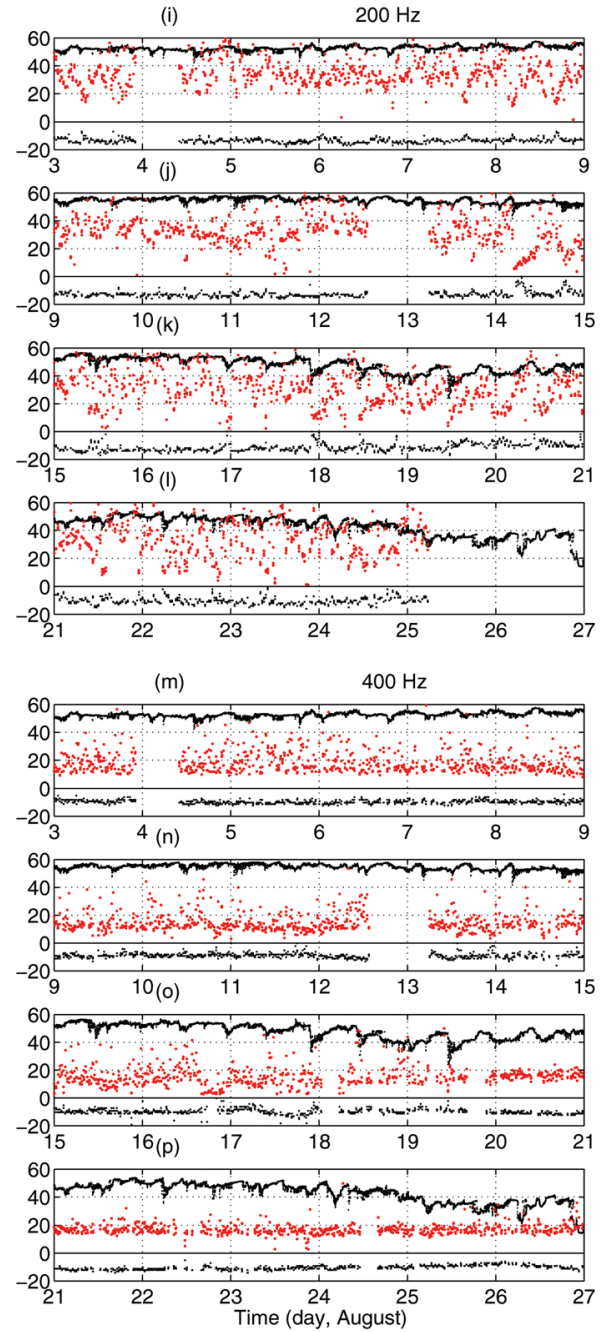

FIG. 4. (Color online) Coherence length and beam-steering angle information are shown for each of the four sources, along with internalwave information. (a)-(d) NE 100 Hz. (e)-(h) NW $224 \mathrm{~Hz}$. (i)-(1) NE 200 Hz. (m)-(p) NW 400 Hz. Data are shown in six-day sections. In red (gray): time series of the maximum (over angle) $L_{d} / \lambda$ estimates for each pulse. Below this, at negative values on the $y$-axes, the steering angle $\theta_{\max }$ yielding this maximum is plotted in black, with $\theta_{\max }$ multiplied by 4 and shifted by a constant before plotting. At the tops of the panels (black) are shifted plots of the $15^{\circ} \mathrm{C}$ isotherm height time series (meters) from sensors at the HVLA. Dips to lower value indicate arrival of internal waves of depression. The numerical labels on the $y$ axes pertain to the nondimensional $L_{d} / \lambda$ values, the (shifted) isotherm height in meters, and the $4 \theta_{\max }$ values in degrees. from which statistics $R, R_{N}$, and $L_{d}$ can be computed. For a bottom-resting array, $z$ is the water depth. For the nominally meridional SW06 HVLA, $\Delta \eta=\cos \theta_{i}\left(x-x_{0}\right), i=1$ to 4 . A diagram of this appears in Collis et al. (2008). A related procedure has been used to propagate a VLA received field into a range-dependent environment (Heaney, 2009). Significantly shorter $L_{d}$ for measured fields versus synthetic fields $\left(p_{s}\right)$ implies azimuthal variation of modal structure. This can be caused by adiabatic mode refraction or azimuthallydependent mode coupling.

\section{CORRELATION RESULTS}

\section{A. Time series of horizontal coherence length}

In this section, horizontal coherence length $\left(L_{d}\right)$ results are reported for the NE and NW sources for a three-week period. Initial work on a small portion of the $100-\mathrm{Hz} \mathrm{NE}$ receptions (three six-hour time windows) uncovered different classes of coherence during differing ocean conditions (Collis et al., 2008). Substantially more data are presented here.

For each pulse, the correlation scale is computed for a collection of beam steering angles $\theta$ spanning $15^{\circ}$ centered at the nominal incident angle for each source $\theta_{i}$. Beam steering angle has a strong effect on $R_{N}(\Delta x)$ and $L_{d}$ for all of our signals, as expected for near plane-wave arrivals (Fig. 3). The maximum $L_{d}$ value is established and then compared with environmental measurements to study processes that cause azimuthal variations in signal (i.e., reduction of $L_{d}$ ).

Time series of individual pulse coherence length estimates for the four sources are plotted in Fig. 4. Values for three matched-filtered pulses are shown for each sequence train (they vary gradually over the sequence trains of a few minutes length). One quantity shown is time series of maximum $L_{d}$ (with respect to $\theta$ ) for each pulse. The angle $\theta_{\max }$ having maximum $L_{d}$ is also shown for each pulse (in the non-physical negative $L_{d}$ space). Finally, time series of $15^{\circ} \mathrm{C}$ isotherms at the HVLA are plotted at the top of each panel, showing internal-wave activity. The direction $\theta_{\max }$ fluctuates in time (beam wobble), as does the coherence length at that steering angle. This is difficult to see in Fig. 4, and is shown more clearly for a short time interval in Fig. 5. The coherence properties fluctuate strongly as internal waves pass over the receiver, over the source, or between the two, as will be shown in more detail later. For these data, the average is over lag realization (sub-aperture average), with each pulse yielding an $R$-curve estimate and an $L_{d}$ estimate. The number of products $\psi(x) \psi^{*}(x+n d x)$ averaged together to form $R(n d x)=\left\langle\psi(x) \psi^{*}(x+n d x)\right\rangle$ for each pulse is $32-n$, where $d x=15 \mathrm{~m}$ for this HLA. Thus, there are greater 
(a) Isotherm displacements at three stations.

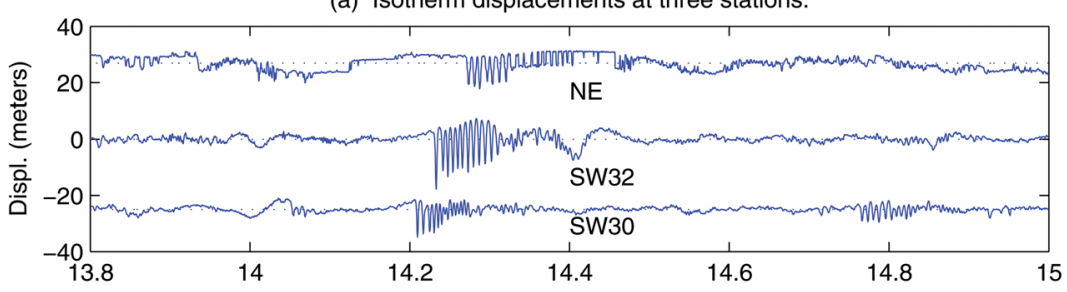

(b) Angle of max $L_{d} \cdot 200 \mathrm{~Hz}$ data.

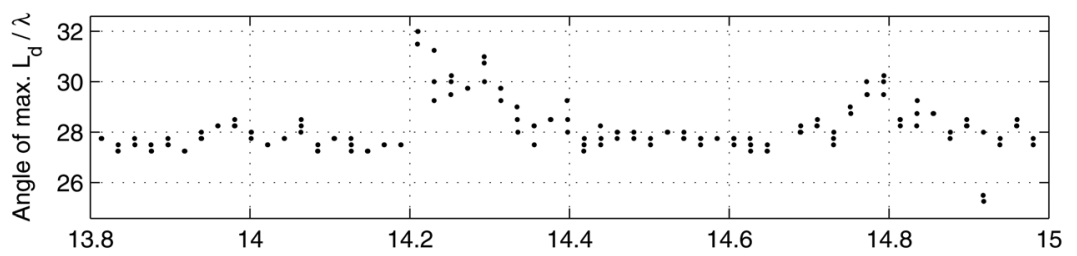

(c) $L_{d}$ values. Red box: Max, over angle. Blue: at fixed angle (27.6 deg)

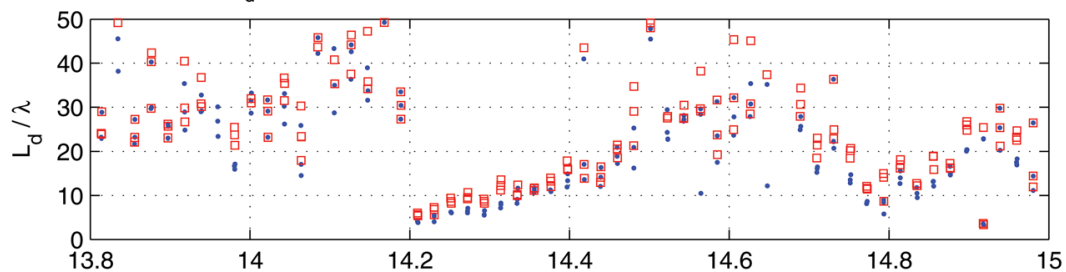

(d) $L_{d}(\theta, t) / \lambda$

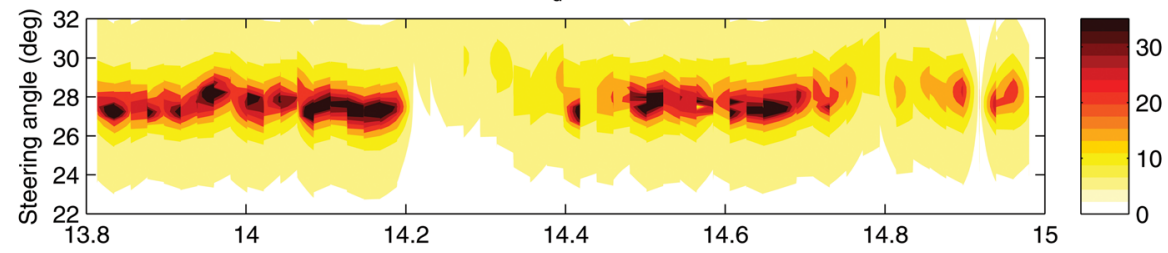

(e) Max HBP, VLA Power, V Mode Powers

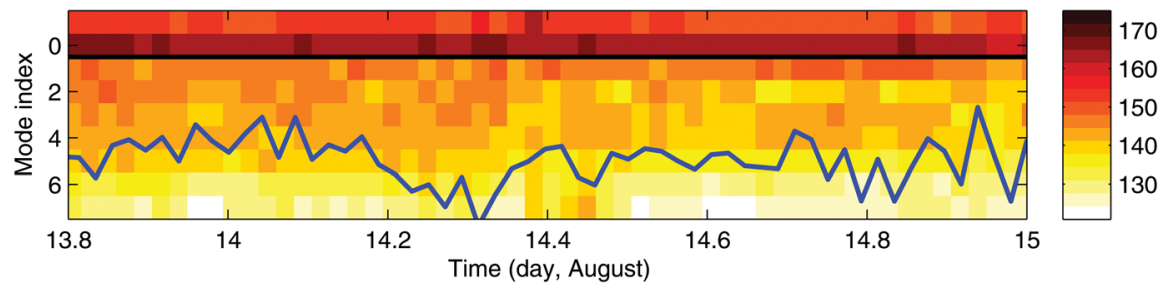

FIG. 5. (Color online) Thermocline displacements and correlation length information for $200-\mathrm{Hz} \mathrm{NE}$ transmissions are shown for 1.2 days. (a) Displacements are shown for three NE-HVLA positions (SW30 is near HVLA). At NE, thermometers provide the $17^{\circ}$ isotherm excursions. 20-m depth vertical velocities from ADCPs are integrated to provide displacement at the other two locations. The waves are seen to reach the receiver before reaching the NE source. (b) The steering angle giving maximum $L_{d}$ is shown. (c) The value of maximum $L_{d}$ (over steering angle) and $L_{d}$ at fixed angle $27.6^{\circ}$ are plotted, with $L_{d}$ normalized by acoustic wavelength $\lambda$. (d) $L_{d} / \lambda$ over steering angle and time is contoured. (e) Power detected in normal modes one to seven at the VLA is plotted. The pulseaverage and depth average of pressure squared at the VLA is also plotted at the axis position for mode zero. The maximum (over steering angle) of horizontal beam power at the HLA is also plotted at the mode -1 position. The line shows an estimate over time of the number of excited modes: the sum of mode power times mode number, divided by the sum of the mode powers.

than 20 degrees of freedom at the smaller lags even for individual pulses, and the error bars for $R$ and $R_{N}$ are very small at short lag. Therefore, pulse-based $L_{d}$ estimates (from $R_{N}(\Delta x)$ ) that are less than half the HLA length are also very reliable, within $10 \%$. The HLA shift of August 19th causes the mean apparent angle of incidence to shift. No attempt was made to perturb $V(x, \theta)$ to bring the pre- and post-event mean angles into agreement.

The variation of $\theta_{\max }$ seen in Fig. 4 is equivalent to a wobble of beam direction with maximum beam power (not shown). Steering in direction fixed in time, either along the geodesic to the source or in an angle determined by maximum coherence at one time, will not always yield the maximum $L_{d}$ (and also maximum beam power), so coherence measurements made in that manner would be biased downward. Figure 5 shows this in detail for two wave events using 200-Hz data.

In the first half of the record, the $100-\mathrm{Hz} L_{d}$ values are 25 to $30 \lambda$, dropping to about $20 \lambda$ for the second half [Figs. 4(a)-4(d)]. The $100-\mathrm{Hz}$ analysis is aperture limited.

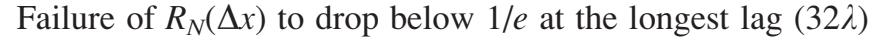
means that the $L_{d}$ is not well measured. These instances are indicated by $L_{d}=32 \lambda$. (Most values are less than this.) Strong short-term variations that appear to be quasi-tidal in period and linked to internal waves (i.e., not random) exist throughout the record, to be addressed in Sec. VI.

The correlation-length studies for the NW sources [Figs. $4(\mathrm{e})-4(\mathrm{~h})$ and $4(\mathrm{~m})-4(\mathrm{p})$ ] show time variation of $\theta_{\max }$, but there is a difference of about $5^{\circ}$ for the pair that is unexplained. Median coherence lengths for each NW source are about $15 \lambda$, lower than for the NE sources. Individual NW $L_{d}$ estimates vary greatly, however, from $5 \lambda$ to $40 \lambda$.

Figure 6 shows details of the behavior of the functions $D(\Delta x, \theta)$, and $R_{N}(\Delta x, \theta)$ for two individual $200-\mathrm{Hz}$ pulses, one with long $L_{d}$ and one with short $L_{d}$. The phase structure functions are computed here as the mean of the squared phase difference of the spatially lagged series $D(\Delta x)=$ $\left[1 /\left(t_{2}-t_{1}\right)\right] \int_{t_{1}}^{t_{2}}(\phi(x)-\phi(x+\Delta x))^{2} d t$. The $D$ and $R_{N}$ functions behave inversely, as expected, with $R_{N}$ high when $D$ is low. This figure also illustrates individual traces of $D(\Delta x)$, 
(a) $\mathrm{D}$

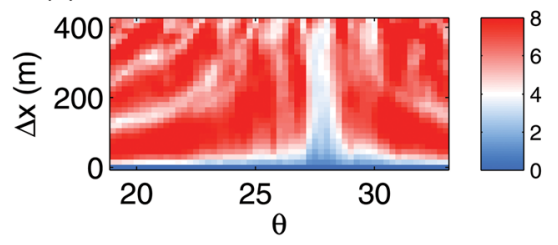

(b) real( $\mathrm{R})$

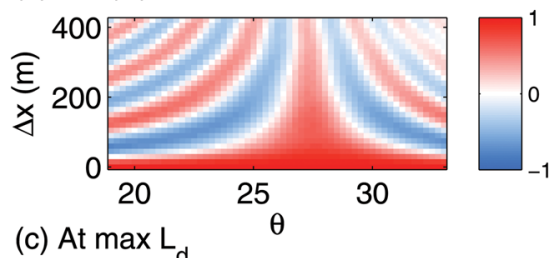

(c) At $\max L_{d}$

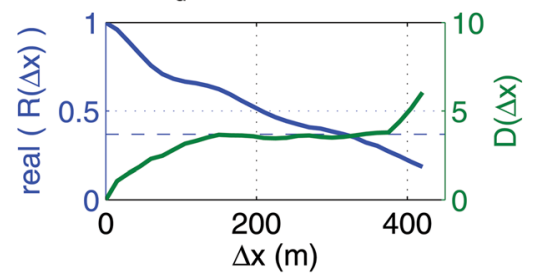

(d) $\mathrm{D}$

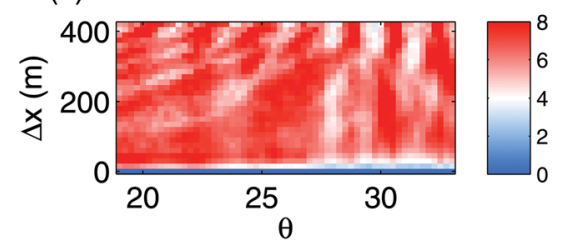

(e) real $(\mathrm{R})$
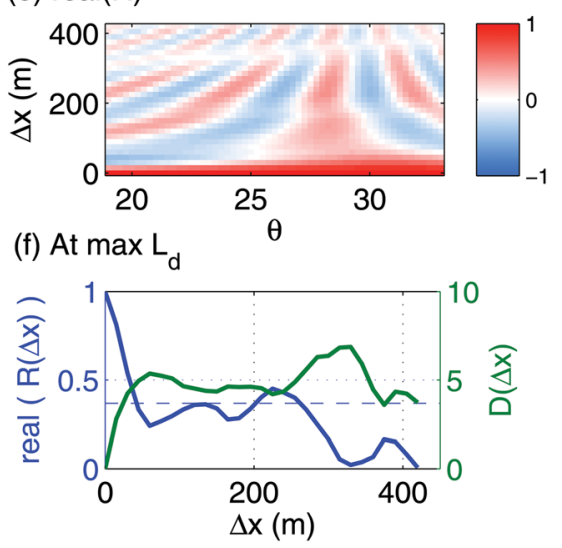

FIG. 6. (Color online) Individual-pulse statistics are plotted for two $200-\mathrm{Hz}$ transmissions [one pulse from the pulse sequence at $14 \mathrm{Au}-$ gust 02:01 UTC (a)-(c), and one at 05:31 on the same day (d)-(f)]. At the top (a),(d) are the phase structure functions $D(\Delta x, \theta)$, in the center (b),(e) are the lag-averaged (but not time-averaged) $R(\Delta x, \theta)$, and at the bottom (c),(f) are the functions $D\left(\Delta x, \theta_{M}\right)$ and $R\left(\Delta x, \theta_{M}\right)$ at the angle of maximum $L_{d}$. and $R_{N}(\Delta x)$, drawn for the steering direction with maximum coherence.

Figure 7 shows ensemble-average $L_{d}$ values for the two NE data sets and one NW data set. These are computed from $R_{N}(\Delta x)$ functions computed in 24- and 3-h time windows for the NE data, and only in 24-h windows for the NW data. For both window sizes, 100 - and $200-\mathrm{Hz} L_{d} / \lambda$ values are approximately equal over the first half of the record. In the second half of the record the $200-\mathrm{Hz} L_{d} / \lambda$ values are longer. Equivalently, in distance units, shown here for 3-h windows only, the $100-\mathrm{Hz} L_{d}$ are generally longer in the first half of the record, and the two $L_{d}$ series are similar in the second half. This similarity in actual scale suggests that the scattered
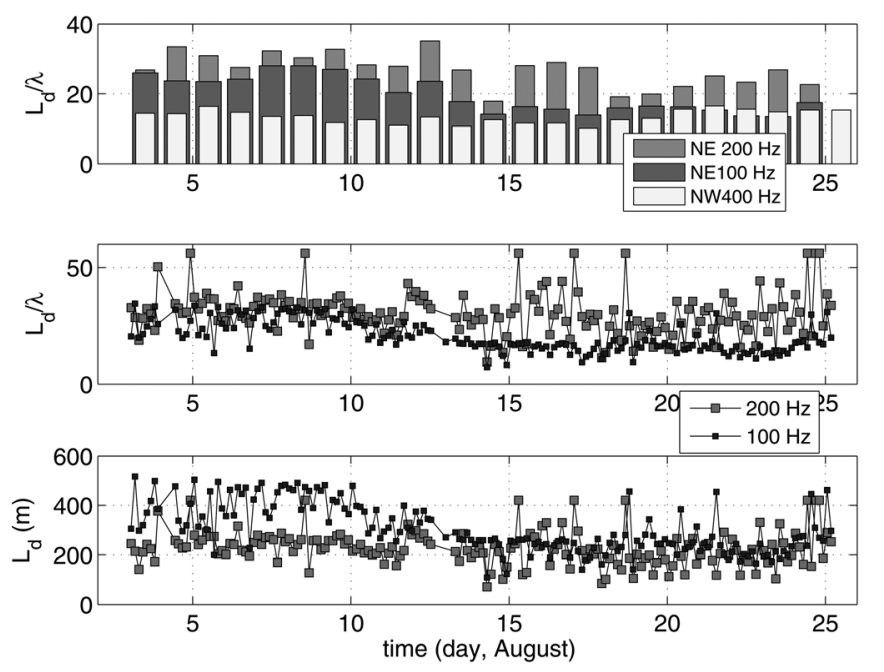

FIG. 7. Maximum (over steering angle) coherence length estimates $\left(L_{d}\right)$ are shown for $100-\mathrm{Hz}$ and $200-\mathrm{Hz}$ NE data and $400-\mathrm{Hz}$ NW data. Two types of time averages are used to compute $R(\theta, \Delta x)$, from which $L_{d}(\theta)$ is computed, of which the maximum is plotted. (a) 24 -h averaging is used, with daily $L_{d}$ resulting, plotted in nondimensional form using the acoustic wavelength. (b) For NE sources, the same quantities as in (a) are shown, although with 3-h averaging is used. (c) The NE-source maximum $L_{d}$ values shown in (b) are plotted again in dimensional form. fields are geometrically similar for the two frequencies, and are not consistent with an interference pattern for a low number of quasi-plane waves. The two NW sources have similar 24-h $L_{d}$ behavior, so only one is shown. These show $L_{d}$ weakly varying between 11 and 16 wavelengths, significantly shorter and steadier than the NE values. The averaged measurements have hundreds of degrees of freedom and thus would have very small error bars under stationary conditions. Of course conditions change, one finding in this paper, so this assumption may not be true and thus the estimated error bars of less than $\pm 10 \%$ may be optimistic. The variations seen in the time series of Fig. 7 appear to reflect real changes in the pulse behavior and do not behave like undersampled randomly varying estimates of a stationary process. This is most true for the NW data with short $L_{d}$, which are estimated exclusively with the high degree of freedom low$\Delta x$ zones of the $R_{N}(\Delta x)$ curves.

\section{B. Horizontal array gain}

Figure 8 shows $G(M)$ for the two NE data sets and the two NW data sets. The upper line is the theoretical maximum array gain, $M$ [this is $10 \log _{10}(M)$ in $\mathrm{dB}$ ]. [The power gain of a perfectly coherent signal is $M^{2}$, the power gain of a complex joint-normal random noise phasor is $M$, and the ratio of these is the theoretical maximum array gain, $G(M)=M$.] With the exception of the $224-\mathrm{Hz}$ data, the gain is reasonably close to the theoretical limit. However, these may be misleading values for two reasons. First, the estimated gain could be positively biased because of data selection; the maximum gain of each pulse (versus angle) is selected. Signal power and noise power are random variables, and thus $G$ is also, and the selection gives extra weighting to high- $G$ realizations. Section VII C shows time-mean $G$ estimates that do not perform this selection over angle, which are lower. Secondly, the noise gain may be less than theoretical. This would be the situation for noise that is 


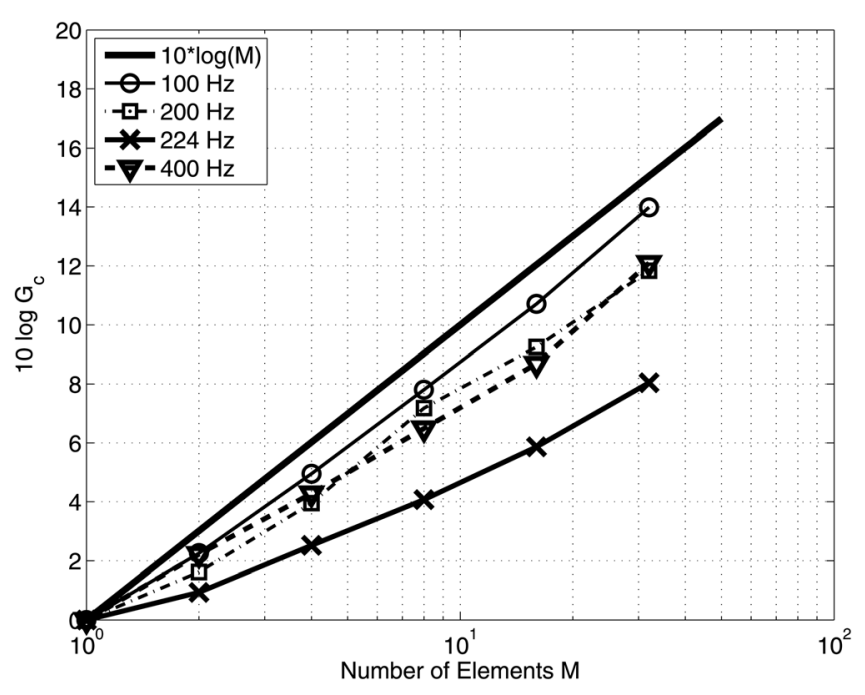

FIG. 8. Array signal gains are shown, averaged over the entire 22 days, for the four source frequencies.

directional, but arriving from a different direction than the signal. Full individual analysis of the signal and noise gains to verify this remains to be done.

\section{Horizontal correlation scales of modal subsets}

Sound propagating from the NE sources to the HVLA undergoes typical shallow-water normal mode dispersion. In addition, specific mode arrivals sometimes show multiple peaks (mode multipath). Collis et al. (2008) show two examples of this in the NE 100-Hz VLA data. Other examples appear in Headrick et al. (2000a) and Lin et al. (2009). Generally mode one arrives first, followed by the initial arrivals of the other modes overlapped with secondary arrival of prior modes. Occasionally modal arrivals are dispersed sufficiently to be non-overlapping in time, thus their behavior can be individually analyzed on the HLA. The timeaveraging interval of the broadband covariance estimates can be long enough to include all arrivals of all modes (i.e., the entire pulse duration), or can be shorter to capture subsets of modes. For example, a short interval at the beginning of the pulse will primarily capture the behavior of mode 1 .

The relationship of mode content to intra-pulse delay time was established for the week of August 14th through the 21 st by analyzing VLA and HLA data. Using this information to adjust window lengths, the spatial correlation behavior of various modes is examined. Estimates of $L_{d}$ from the week-long ensemble averages are shown in Fig. 9 for $100 \mathrm{~Hz}(\mathrm{NE})$ and for various window lengths (i.e., various modal subsets). In the top panel, $L_{d}$ estimates are shown for window durations corresponding to the first mode, the first and second modes, modes one through four, and finally for the entire pulse duration. The bottom panel shows a similar analysis, but instead uses sequentially longer windows. The window lengths are the initial $0.1 \mathrm{~s}$ of the pulse, the initial $0.2 \mathrm{~s}$ of the pulse, and so on. Two regimes of coherence length are evident in the plots. The low-order modes are coherent beyond the length of the array, but the higher order modes that arrive later are not. The higher-order modes have
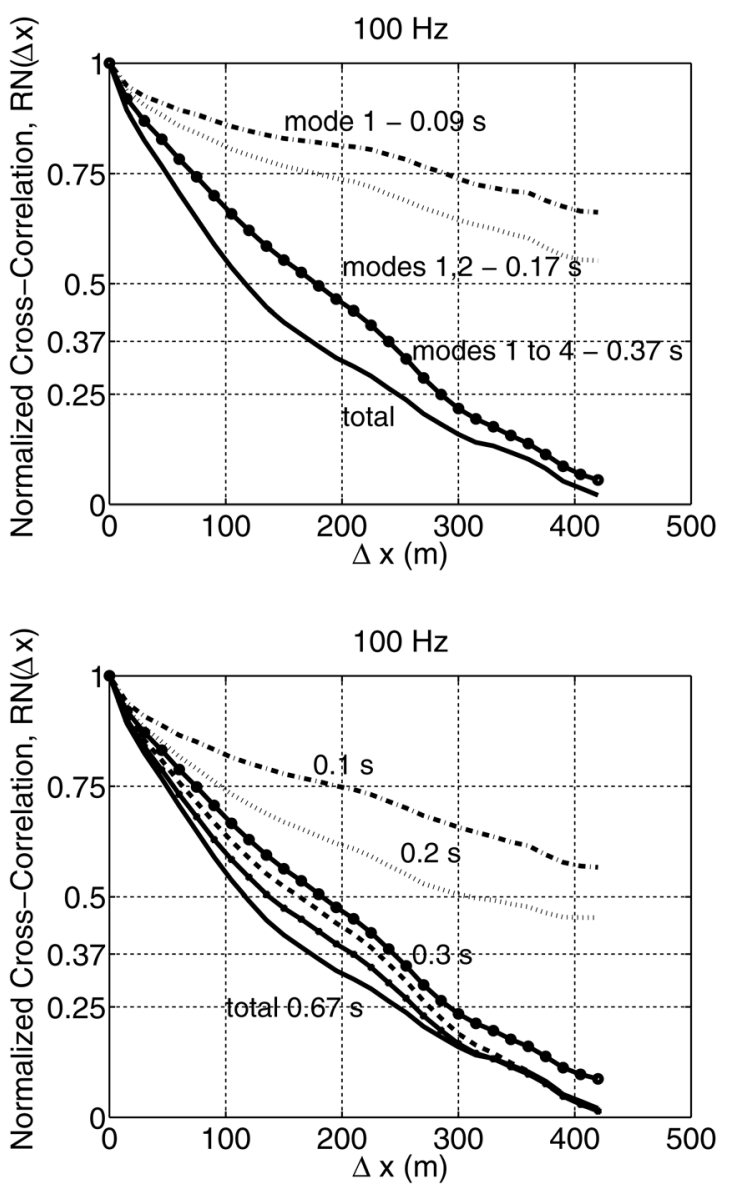

FIG. 9. Functions $R_{N}(\Delta x)$ are shown for the one-week period Aug 14-21. Top: Values computed using signals only from specific time subintervals of the pulse arrivals corresponding to arrivals of specific modes detected with the VLA are analyzed. Data from the leading edge of the pulse arrivals to the times indicated are shown. Bottom: In similar fashion, values computed using data only from time subintervals determined objectively are shown; again, subintervals extend from the leading edge to the times indicated. Early arrivals (low-mode arrivals) maintain higher correlation value at long lag. Values are calculated for six intervals of lengths $0.1,0.2,0.3,0.4,0.5$, and $0.67 \mathrm{~s}$, appearing in that sequence in the figure. The sixth interval includes the entire pulse arrival.

sufficient energy to reduce overall pulse $L_{d}$ values to order $12 \lambda(180 \mathrm{~m})$.

\section{Measured-pulse and synthetic-pulse $L_{d}$}

Data of 14 August are used to verify the azimuthal variability of the modal content of the pulses. Pulses consisting of cylindrically-spreading normal modes (close to plane waves for this experiment) will show spatial decorrelation at oblique angle because the modal interference pattern is a function of range. The familiar image of sound energy versus range at fixed depth from parabolic equation simulations illustrates this. The scale $L_{d}$ for this situation is computed using the synthetic version for each pulse, identically as for the actual measured pulses. Figure 10 shows synthetic-pulse and actual-pulse $L_{d} / \lambda$ computed with 2.4 -h time averaging for three sources. The coherence lengths are much longer for the synthetic pulses. Thus, trivial along-array changes of the modal interference pattern are not responsible for the HLA data-based correlation scale values. The implication is that 

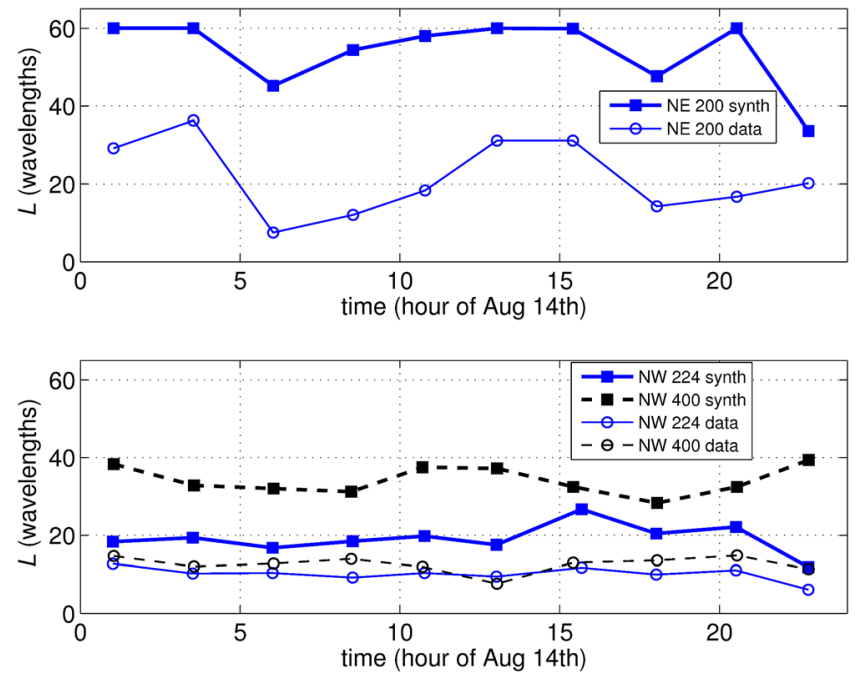

FIG. 10. (Color online) 2.4-h window-averaged correlation lengths (1/e points) are shown, obtained for ten windows on 14 August. Values are shown for both measured HLA pulses and synthetic HLA pulses. Top: NE source results; bottom: NW source results. To normalize, the correlations lengths for each acoustic frequency $(200,224,400 \mathrm{~Hz})$ are divided by the wavelength. The measured-pulse results are obtained by computing $R_{N}(\Delta x)$ by cross-product averaging over pulses in each window and over duplicate element spacing $\Delta x$. The synthetic results are obtained using pulses computed along a due-north straight array using expression (8) and VLA data. Many of the synthetic $L$ estimates are greater than the array length, in these cases underestimates roughly equal to the array length are given.

the pulses measured at the HLA contain true azimuthal variability.

\section{DETAILS OF HORIZONTAL COHERENCE TEMPORAL VARIATION}

\section{A. Wave-packet effects}

Time series of $L_{d}$ estimates and the relationship between coherence changes and intermittent internal-wave activity are investigated in more detail in this section. Figure 5 clearly shows the association of an internal-wave packet passing over the NE to HVLA sound path with rapid $200-\mathrm{Hz}$ signal $L_{d}$ fluctuations and rapid arrival-angle fluctuations. The packet is seen to span the path, but passing at an angle, arriving first at the HVLA and last at the NE sources. The isotherm excursions shown in the figure are up to $20 \mathrm{~m}$ in size in 80-m deep water, typical of energetic nonlinear internal waves (Apel et al., 1997).

Figure 5 shows $L_{d}$ values computed on a pulse-by-pulse basis, along with HLA beam power time series, VLA power time series, and modal arrival energies at the VLA. Plotted in the fourth panel in contour fashion are $L_{d}$ values computed at many steering angles for each pulse (at each time). The steering angle giving maximum $L_{d}$ at each time is plotted, as are the associated maximum $L_{d}$ values. The maximum $L_{d}$ falls abruptly as the waves pass initially by the HVLA and then into the propagation path over the course of about an hour at August 14.21 (14 August 05:00 UTC). At the same time the angle of maximum $L_{d}$ rises, so that the energy appears to arrive from a more easterly direction by up to $4^{\circ}$. This is in agreement with refraction to the right (looking from the source) of propagating sound encountering, at the left, the leading internal wave of anomalously high modal phase velocities. One semidiurnal tidal period later, near time 14.8, a set of smaller amplitude internal waves produces a similar effect that is weaker and not as abrupt in time. The lowest panel of Fig. 5 shows that the energy is less restricted to lower modes at the time of internal-wave passage, with the number of energized modes rising to over seven from a background level of about five. The effect precedes the time of packet arrival at the source NE (time $~ 14.27$ ). This suggests a change in mode coupling behavior. Maximum HLA beam power (maximum over steering angle) and VLA energy fluctuate throughout the record, not simply in concert with wave packets. However, dominant $R_{N}(\Delta x, \theta)$ and $L_{d}$ fluctuations are linked with packets.

The pulse-by-pulse $L_{d}$ estimates shown in Figs. 4 and 5 have limited degrees of freedom and ad-hoc error bars of order $\pm 20 \%$. The daily-average $L_{d}$ results obtained with one-day averaging of lagged field products (Fig. 7) are more reliable but do not capture the rapid changes seen in the pulse-by-pulse values. The 3 -h average values also shown in Fig. 7 have very small error bars, but the averaging window boundaries are not well matched to the internal-wave events. A moving window computation would be best. As a compromise, Fig. 10 shows very reliable $L_{d}$ estimates for 14 August made with 2.4-h windows that are well matched to the wave events. The 200-Hz results for window 3 (times 14.2 to 14.3, measured in days) of Fig. 10 show the steep drop in fielddata $L_{d}$ compared to the earlier intervals. This is in close agreement with the Fig. $5 L_{d}$ estimates, but with tighter confidence intervals. The agreement between the individualpulse estimates and the long temporal averages show that the individual-pulse estimates are quite robust.

$\backslash$ The high-reliability $L_{d}$ estimates of Fig. 10 show with confidence that the NE-source correlation behavior is strongly affected by the waves, whereas the NW-source behavior is not. Coherence lengths in the along-shelf (NE) direction are three to four times those in the across-shelf (NW) direction between wave events, but drop to about the same level for the two directions when the waves occupy the NE path. Reduced NW $L_{d}$ can be attributed to the NW to NE propagation distance ratio of 1.5. They are also associated with propagation that is generally orthogonal to ever-present internal-wave fronts, expected to show strong mode coupling. The observed short correlation lengths for NW are less than obtained with a simple model of coupled-mode propagation through straight-crested uniform internal waves (Duda, 2006), suggesting that the simple model does not adequately describe the situation. A candidate explanation is that waves have along-crest variability, and thus mode coupling has along-crest variability, adding to the mode phase variability that dominates in the simple model.

\section{B. Gain analysis and beam wobble}

The change in $L_{d}$ of $200-\mathrm{Hz}$ signals $4.8 \mathrm{~h}$ into August 14th seen in Fig. 5 (at time 14.2) has a strong effect on array gain. Figure 11 shows array gain degradation (AGD.M) for $M=4$ and $M=32$ elements for two 2.4-h time periods, day 14.0 to 
(a)

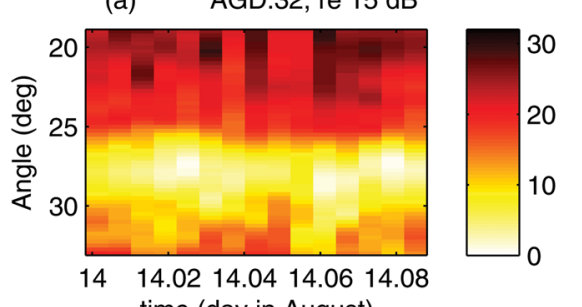

time (day in August)

(b)

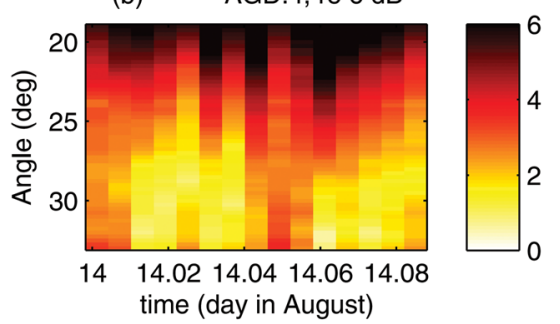

(c)

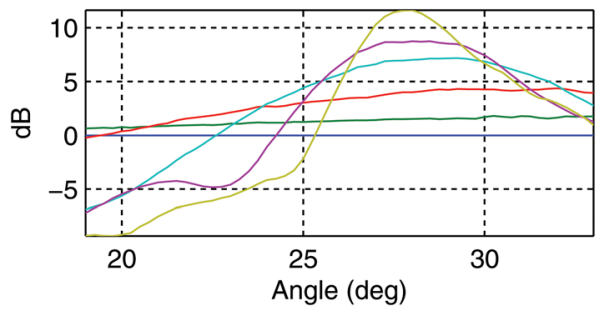

(d)
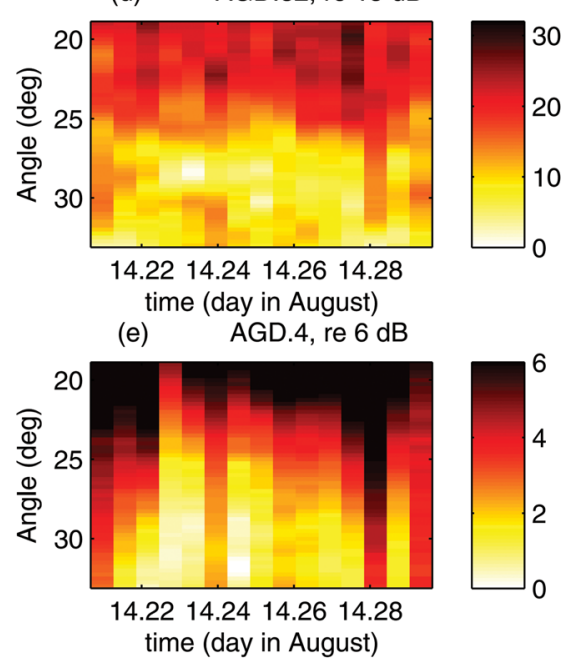

(f) Window-mean gain

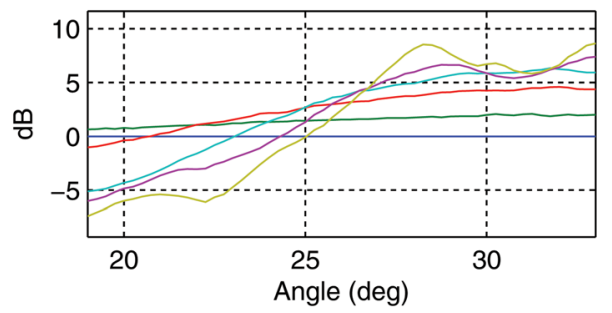

FIG. 11. (Color online) 200-Hz (NE) array gain degradation (AGD) (a), (b), (d), (e) and array gain (c), (f) are shown, all in $\mathrm{dB}$ units. Results are from two 2.4-h long windows on 14 August, hours 0-2.4 [left, (a)-(c)] and hours 4.8-7.2 [right, (d)-(f)]. (a) and (d) show full-array AGD results (32 elements at $15-\mathrm{m}$ spacing) for all analyzed pulses in each time window, with the time scale distorted (long gaps not shown), for a fan of steering angles. White indicates zero array gain degradation (theoretical SNR gain of $15 \mathrm{~dB}$ achieved). (b) and (e) show four-element subarray results in a similar format as the 32-element results (theoretical gain is $6 \mathrm{~dB}$ ). Results are averaged over the multiple short subarrays. (c) and (f) show window-mean gain at each setting angle, converted to $\mathrm{dB}$ after averaging. Subarray lengths are one element (flat line, no gain), 2, 4, 8, 16, and 32 elements. The highest gain is achieved with 32 elements; the gain increases montonically with subarray length near $28^{\circ}$ for each window, indicating line coloration.
14.1 and day 14.2 to 14.3 . Quantity AGD.M is theoretical gain (in $\mathrm{dB}$ ) minus achieved gain (in $\mathrm{dB}$ ) for an $M$-element array, AGD.M $=10\left(\log _{10} M-\log _{10} G\right)$. Degradation for numerous pulses is plotted versus steering angle in the four upper panels. In the first interval there is a broad peak of good performance near $28^{\circ}$ for $M=32$ [light color, low AGD, Fig. 11(a)]. Performance is comparably good for $M=4$ [Fig. 11(b)]. In the later period of strong internal waves and reduced $L_{d}$ the gain performance is degraded [Figs. 11(d) and 11(e)].

Averaging over the fluctuating performance in each window may give a more meaningful result. Below panels (a) and (b), Fig. 11(c) shows the 2.4-h window mean gains $G(M)$ for all subarray sizes $(M=1,2,4,8,16,32)$. Figure 11(f) shows the same thing for the later interval. The early interval shows a relatively narrow peak of good performance for 32element gain at the direction towards the source, 11 versus $15 \mathrm{~dB}$ theoretical (ideal) performance. Performance peaks in this direction for $M$ of 8,16 , and 32. The later interval shows decreased gain performance at all these high $M$ values. Gains for these values also have wider peaks (wider in angle) or multiple peaks. The $M=8$ peak has shifted to near $32^{\circ}$, up from $29^{\circ}$ in the earlier period. This corresponds to the shift in the angle of maximum $L_{d}$ (Fig. 5). Finally, two peaks of high gain can be seen for $M=16$ and for $M=32$ in the later period [Fig. 11(f)]. This is consistent with two arriving wavefonts, one direct and one at higher angle (to the right looking outward from the HLA). The second wavefront is consistent with refraction from the front side of one or more of the westward moving internal waves in the packet traversing the area (with crests roughly aligned with the sound path). The waves have anomalously high acoustic mode phase speeds, allowing this refraction to occur, deflecting acoustic modes in a manner resembling a reflection (Lynch et al., 2006; Badiey et al., 2011).

\section{SUMMARY}

Observations of strongly time-varying spatial coherence properties of broadband sound propagated $19 \mathrm{~km}$ and $30 \mathrm{~km}$ through intermittent internal waves on a continental shelf have been examined. Sound was recorded with a bottom-resting horizontal line array with a vertical line array attached at one end. This receiver was moored in water of about $80 \mathrm{~m}$ depth.

Techniques for estimating spatial coherence tailored to these data have been explained and used here. The resulting estimates of broadband horizontal spatial-lag correlation functions $R_{N}(\Delta x)$ and the point $\Delta x=L_{d}$ where these descended to the value $1 / e$ (from one at $\Delta x=0$ ) were highly reliable for short time intervals. The reasons for this were high signal to noise ratio (enabled by coded pulse compression), an ability to determine array geometry from dispersed mode arrivals and therefore an ability to correct phase, and many repeated lags at the scale $\Delta x$ where $R_{N}(\Delta x)$ approached $1 / e$. Coherence length $L_{d}$ and coherent array gain (signal to noise ratio improvement) are the main variables examined here. There are many key results.

Coherence behavior is strongly anisotropic. Along wave crest and across wave crest sound propagation differs in temporal variability of $L_{d}$ and mean $L_{d}$. Sound from the $30-\mathrm{km}$ distant NW sources traveling in the same direction as internal waves (across crest) had short $L_{d}$ that was steady in time, approximately 10 to 15 acoustic wavelengths for both 224 and $400 \mathrm{~Hz}$ (Figs. 4, 7, and 10). Next, sound from 19-km distant NE sources at 100 and $200 \mathrm{~Hz}$ traveling perpendicular to the 
internal-wave propagation direction (i.e., along the internalwave crests) had highly variable $L_{d}$ for short-time windowing of $3 \mathrm{~h}$ or less (Figs. 4, 7, and 10). As internal waves passed, the correlation scale dropped from approximately 25 to 7 wavelengths at $100 \mathrm{~Hz}$, and from approximately 40 to 7 wavelengths at $200 \mathrm{~Hz}$ (Figs. 4 and 5, 7, and 10).

When longer-term averages over one-day windows are considered, the $L_{d}$ estimates for the NE-source sound are stable over time. During the first 10 days of the experiment the 100 and $200-\mathrm{Hz} L_{d}$ are similar when scaled by wavenumber (i.e., when $L_{d} / \lambda$ is considered), with both fluctuating weakly around 23 to 30 wavelengths (Fig. 7). This scaling with wavelength is consistent with an interference pattern from a small number of beams. This is somewhat supported by array gain degradation and array gain results which suggest two beams of coherent energy (Fig. 11). During the latter half of the experiment the $L_{d} / \lambda$ scale for $100-\mathrm{Hz}$ sound drops below that of $200-\mathrm{Hz}$ sound; in dimensionful terms the $L_{d}$ scales are very similar [Fig. 7(c)]. This absence of coherence-length scaling with wavelengths is not consistent with an interference pattern from a small number of beams developing for each frequency, but instead suggests some type of divided-beam geometry that is spatially similar for the two frequencies and may be closely tied to internal-wave mode-duct geometry. Images of such beams can be found in at least two papers (Lin et al., 2009 and Lynch et al., 2010), but the beam geometry versus frequency has not yet been examined in detail. There may be other explanations.

The low-order modes that arrive first in the pulses are much more coherent than the later modes for the $100-\mathrm{Hz}$ signals (Fig. 9). In fact, the early modes are highly coherent for the entire array length of $465 \mathrm{~m}$. The later modes have short $L_{d}$ and carry sufficient energy to allow their behavior to reduce the overall coherence of the pulses.

Phase structure functions and correlation functions are complementary, as expected (Fig. 6). This figure also shows $R_{N}(\Delta x)$ function shapes for a high- $L_{d}$ prior to the arrival of an internal-wave packet at the acoustic path, and a low- $L_{d}$ situation during the presence of the packet.

Computation of $L_{d}$ for synthetic fixed-mode pulses consistent with the VLA signals at 224, 200, and $400 \mathrm{~Hz}$ quantify the degree of decorrelation expected from the departure from broadside of the signal arriving at the HLA (i.e., the oblique incidence). These are longer than observed in the data (Fig. 10), indicating a departure of the oceanic signals from azimuthal uniformity in mode content. Such pulse structure may be caused by azimuthally-dependent mode coupling, or by refracted adiabatic mode propagation.

This paper includes only a subset of the information available from this experiment. Further analysis of the pulse behavior may answer some open questions. For instance, what is the cause of the change in scaling behavior versus wavelength halfway through the experiment (Fig. 7)? Also, why are the NE-path statistics so stable despite the change in internal-wave behavior over time (depicted in Fig. 4) that has such strong effect on the NE path $L_{d}$ ? Is the expected coupled-mode propagation from NW truly always saturated at this range, and if so, at what ranges does this hold? Finally, what will the behavior be at other path angles with respect to internal-wave crests, and at other source-receiver distances?

\section{ACKNOWLEDGMENTS}

This work was supported by Office of Naval Research (ONR) Grants Nos. N00014-05-1-0482 and N00014-11-10194 to T.F.D., ONR Grant No. N00014-04-1-0146 to J.F.L., and an ONR Ocean Acoustics Postdoctoral Fellowship awarded to J.M.C. under Professor William Carey at Boston University. The moored instruments providing the data used here were placed by $R V$ Knorr of Woods Hole Oceanographic Institution (WHOI). The HVLA instrument and the sound sources were recovered by $R V$ Knorr. Other moorings were recovered by $R V$ Oceanus of WHOI. The captains and crews of the vessels are commended. The efforts of John Kemp, Jim Ryder, and Neil McPhee of WHOI in mooring design, deployment, and recovery are acknowledged. The efforts of Keith von der Heydt, Ed Scheer, Steve Liberatore, Jim Irish, Neil Mcphee of WHOI in instrument design, fabrication, and operation are acknowledged. The efforts of Hien Nguyen, Mike Rebozo, and Jennifer Wylie of the University of Miami in sound source preparation and operation are acknowledged.

\section{APPENDIX A: BROADBAND CORRELATION FUNCTION}

The correlation function $C$ of the field $\psi$ is $C\left(x, x^{\prime}, t, t^{\prime}\right)=\left\langle\psi(x, t) \psi^{*}\left(x^{\prime}, t^{\prime}\right)\right\rangle$, where the brackets indicate ensemble average. For stationary signals, $C$ is independent of $x$ and $t$ so that $C\left(x, x^{\prime}, t, t^{\prime}\right)=C(\Delta x, \Delta t)$ for any $x$ and $t$, where displacements $\Delta x=x-x^{\prime}$ and $\Delta t=t-t^{\prime}$.

For a harmonic signal given by $\psi(x, t)=A(x) \exp \left(i \omega_{0} t\right)$ the correlation function at zero time lag is simply $C_{H}(\Delta x, 0)=\left\langle A(x) A^{*}(x+\Delta x)\right\rangle$. Some prior investigations of broadband ocean-acoustic correlation have used complex demodulates of the signal and have analyzed only one frequency, thereby using only a small part of the signal energy (Orr et al., 2004).

For a broadband and spatially dependent signals, use of the Fourier transform pairing $\psi(x, t)=(1 / 2 \pi) \int B(x, \omega) e^{i \omega t} d \omega$ and $B(x, \omega)=\int \psi(x, t) e^{-i \omega t} d t$, and of the Wiener-Khintchine relation, yields broadband correlation functions $R$

$$
R(\Delta x, \Delta t)=\frac{1}{2 \pi} \int\left\langle B(x, \omega) B^{*}(x+\Delta x, \omega)\right\rangle e^{i \omega \Delta t} d \omega .
$$

At zero time delay, i.e., $t=t^{\prime}$, this involves an integral over frequency

$$
R(\Delta x)=\left\langle\frac{1}{2 \pi} \int B(x, \omega) B^{*}(x+\Delta x, \omega) d \omega\right\rangle,
$$

where the ensemble average and the integration have been interchanged. By Parseval's theorem this can be written

$$
R(\Delta x)=\left\langle\int_{-\infty}^{\infty} \psi(x, t) \psi^{*}(x+\Delta x, t) d t\right\rangle
$$


(a)

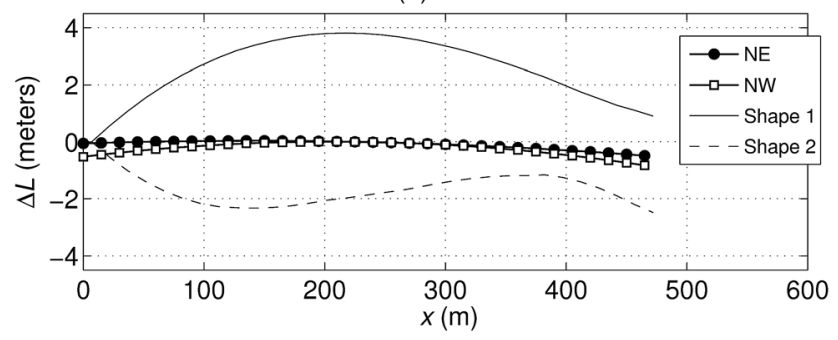

(b)

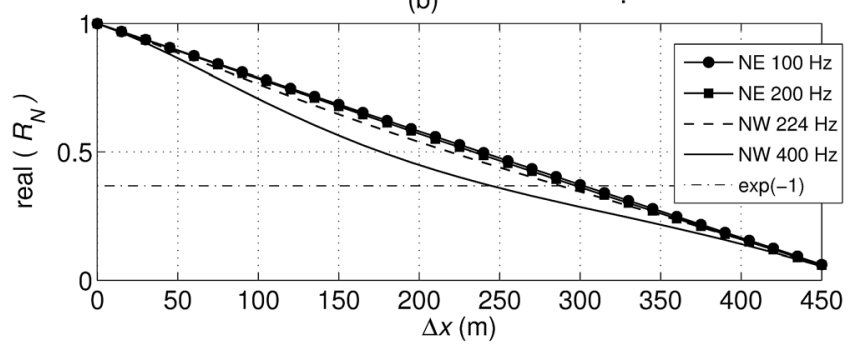

(c)

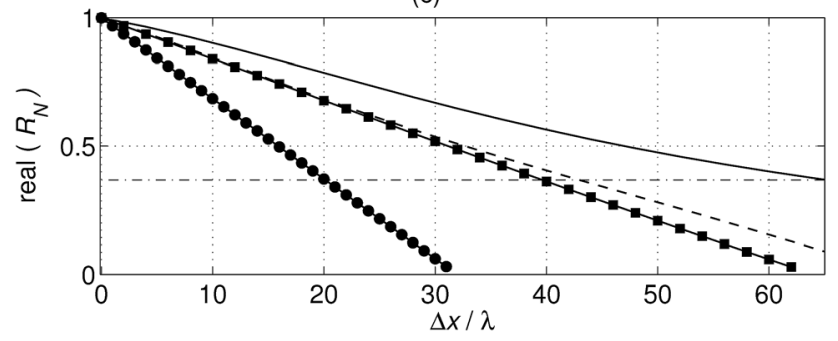

FIG. 12. (a) Range difference $r(x)$ between curved wavefront arrivals from the NE and NW sources and hypothetical plane-wave arrivals, computed as a function of HLA element position (shown with symbols). The other two lines show the NE-path projected array shape anomalies $r_{100}(x)$ before (solid line) and after (dashed line) the wave event of 19 August. (b) The real parts of the $R_{N}$ functions computed for each of the four sources using phase anomalies calculated using the curved wavefronts shown with symbols in (a). (c) The real parts of the $R_{N}$ functions of (b) are shown normalized with acoustic wavenumber. Nondimensional integral scale is shortest for NE 100 Hz. The legend in (b) holds for (b) and (c).

For a signal of finite duration that exists only for $t_{1} \leq t \leq t_{2}$, the integration limits are set to span the signal, giving the approximate correlation function statistic

$$
R(\Delta x)=\left\langle\int_{t_{1}}^{t_{2}} \psi(x, t) \psi^{*}(x+\Delta x, t) d t\right\rangle .
$$

This is the statistic that is analyzed in this paper, with the addition of a normalization factor. The normalized statistic with unity value at zero spatial lag is

$$
R_{N}(\Delta x)=\left\langle\frac{\int_{t_{1}}^{t_{2}} \psi(x, t) \psi^{*}(x+\Delta x, t) d t}{\left(\int_{t_{1}}^{t_{2}}|\psi(x, t)|^{2} d t \int_{t_{1}}^{t_{2}}|\psi(x+\Delta x, t)|^{2} d t\right)^{1 / 2}}\right\rangle .
$$

The procedure is to compute the normalized zero-lag crosscorrelation function for each occurrence of each lag $\Delta x$, producing individual $R_{N}$ ensemble members. These are averaged over duplicated lags along the array and over time (optionally, hours or days), indicated by the brackets.

\section{APPENDIX B: DECORRELATION EFFECT OF CURVED WAVEFRONTS}

Under conditions of cylindrical spreading the arriving wavefronts will have residual phases after beam steering with a plane-wave assumption. In the SW06 area of variable water depth and water-column features such as fronts and eddies the actual mean wavefront geometry is probably more complicated. Nonetheless, the $L_{d}$ scales resulting from the plane-wave beam steering used here for a perfectly coherent but curved wavefront will be computed. The procedures form a guideline for the maximum detectable coherence (or, for reduction of coherence introduced by the processing). Note that the NE signals are processed such that mean curvature is removed, and performance should be better than shown here for those signals.

Figure 12(a) shows the distance anomalies $r(x)$ along the HLA of curved wavefronts arriving from the two sources, which is wavefront departure from a straight line perpendicular to a line connecting the source and the center of the array. The curve shapes are not very sensitive to small departures of the line from this angle. The NE source angle is further from broadside and these sources have lower anomalies because the array projects onto a smaller arc than for NW, despite the relative closeness of NE which imparts greater wavefront curvature. Phase anomalies for the four sources can be computed from the distance anomalies, $\phi(x)=2 \pi r(x) / \lambda$, from which fields $\psi(x)=\exp (i \phi(x))$ are computed. The real parts of the resulting correlations functions $R_{N}(\Delta x)=\left\langle\psi(x) \psi^{*}(x+\Delta x)\right\rangle$ $=\langle\exp (i(\phi(x)-\phi(x+\Delta x))\rangle$ are shown in Fig. 12. The integral scales $L_{d}$ are between 250 and $300 \mathrm{~m}$. In nondimensional form they are greater than $40 \lambda$ except for $\mathrm{NE} 100 \mathrm{~Hz}$, where the value is $20 \lambda$. However, note that the phase adjustment function $V(x, \theta)$ used in beam steering accounts for the detected mean curvature of $100-\mathrm{Hz}$ mode-one signals, so this is an overly pessimistic performance bound.

Apel, J. R., Badiey, M., Chiu, C.-S., Finette, S., Headrick, R., Kemp, J., Lynch, J. F., Newhall, A., Orr, M. H., Pasewark, B. H., Tielbuerger, D., Turgut, A., von der Heydt, K., and Wolf, S. (1997). "An overview of the 1995 SWARM shallow water internal wave acoustic scattering experiment," IEEE J. Oceanic Eng. 22, 465-500.

Badiey, M., Katsnelson, B. G., Lynch, J. F., Pereselkov, S., and Siegmann, W. L. (2005). "Measurement and modeling of three-dimensional sound intensity variations due to shallow-water internal waves," J. Acoust. Soc. Am. 117, 613-625.

Badiey, M., Katsnelson, B. G., Lynch, J. F., and Pereselkov, S. (2007). "Frequency dependence and intensity fluctuations due to shallow water internal waves," J. Acoust. Soc. Am. 122, 747-760.

Badiey, M., Katsnelson, B. G., Lin, Y.-T., and Lynch, J. F. (2011). "Acoustic multipath arrivals in the horizontal plane due to approaching nonlinear internal waves," J. Acoust. Soc. Am. 129, EL141-EL147.

Berkson, J. M. (1980). "Measurements of coherence of sound reflected from ocean sediments," J. Acoust. Soc. Am. 68, 1436-1441.

Carey, W. M. (1998). "The determination of signal coherence length based on signal coherence and gain measurements in deep and shallow water," J. Acoust. Soc. Am. 102, 831-837.

Carey, W. M., and W. B. Moseley "Space-time processing, environmentalacoustic effects,” IEEE J. Oceanic Eng. 16, 285-301 (1991).

Carey, W. M., Lynch, J. F., Siegmann, W. L., Rozenfeld, I., and Sperry, B. J. (2006). "Sound transmission and spatial coherence in selected shallow-water areas: Measurements and theory," J. Comput. Acoust. 14, 265-298. 
Chiu, C.-S., Ramp, S. R., Miller, C. W., Lynch, J. F., Duda, T. F., and Tang, T. Y. (2004). "Acoustic intensity fluctuations induced by South China Sea internal tides and solitons," IEEE J. Oceanic Eng. 29, 1249-1263.

Collis, J. M., Duda, T. F., Lynch, J. F., and DeFerrari, H. A. (2008). "Observed limiting cases of horizontal field coherence and array performance in a time-varying internal wavefield," J. Acoust. Soc. Am. 124, EL97-EL103.

Cox, H. (1973). "Line array performance when the signal coherence is spatially dependent," J. Acoust. Soc. Am. 54, 1743-1746.

Dahl, P. H. (2004). "Forward scattering from the sea surface and the van Cittert-Zernike theorem," J. Acoust. Soc. Am. 115, 589-599.

Dowling, R. D., and Jackson, D. R. (1993). "Coherence of acoustic scattering from a dynamic rough surface," J. Acoust. Soc. Am. 93, 3149-3157.

Duda, T. F. (2004). "Acoustic mode coupling by nonlinear internal wave packets in a shelfbreak front area," IEEE J. Oceanic Eng. 29, 118-125.

Duda, T. F. (2006). "Temporal and cross-range coherence of sound traveling through shallow-water nonlinear internal wave packets," J. Acoust. Soc. Am. 119, 3717-3725.

Duda, T. F. (2007). "Examining the validity of approximations to fully three-dimensional shallow-water acoustic propagation through nonlinear internal gravity waves," in Oceans 2007-Europe Conference Proceedings, Aberdeen (IEEE, New York), pp. 1-5.

Duda, T. F., Flatté, S. M., Colosi, J. A., Cornuelle, B. D., Hildebrand, J. A., Hodgkiss, Jr., W. S., Worcester, P. F., Howe, B. M., Mercer, J. A., and Spindel R. C. (1992). "Measured wavefront fluctuations in 1000-km pulse propagation in the Pacific Ocean," J. Acoust. Soc. Am. 92, 939-955.

Duda, T. F., Lin, Y.-T., and Reeder, D. B. (2011). "Observationally-constrained modeling of sound in curved ocean internal waves: Examination of deep ducting and surface ducting at short range," J. Acoust. Soc. Am. 130, 1173-1187.

Duda, T. F., Lynch, J. F., Newhall, A. E., Wu, L., and Chiu, C.-S. (2004). "Fluctuation of 400-Hz sound intensity in the 2001 ASIAEX South China Sea Experiment," IEEE J. Oceanic Eng. 29, 1264-1279.

Duda, T. F., and Preisig, J. C. (1999). "A modeling study of acoustic propagation through moving shallow-water solitary wave packets," IEEE J. Oceanic Eng. 24, 16-32.

Emery, W. J., and Thomson, R. E. (2001). Data Analysis Methods in Physical Oceanography, 2nd ed. (Elsevier, Amsterdam), Sec. 5.3.

Finette, S., and Oba, R. (2003), "Horizontal array beamforming in an azimuthally anisotropic internal wave field," J. Acoust. Soc. Am. 114, 131-144.

Flatté, S. M. (1983). "Wave propagation through random media: Contributions from ocean acoustics," Proc. IEEE 71, 1267-1294.

Fredricks, A., Colosi, J. A., Lynch, J. F., Gawarkiewicz, G., Chiu, C.-S., and Abbot, P. (2005). "Analysis of multipath scintillations from long range acoustic transmissions on the New England continental slope and shelf," J. Acoust. Soc. Am. 117, 1038-1057.

Frankenthal, S. (1989). "The mutual coherence function in a scattering channel- A two-scale solution," J. Acoust. Soc. Am. 85, 104-113.

Frank, S. D., Badiey, M., Lynch, J. F., and Siegmann, W. L. (2005). "Experimental evidence of three-dimensional acoustic propagation caused by nonlinear internal waves," J. Acoust. Soc. Am. 118, 723-734.

Headrick, R. H., Lynch, J. F., Kemp, J. N., Newhall, A. E., von der Heydt, K., Apel, J., Badiey, M., Chiu, C.-S., Finette, S., Orr, M., Pasewark, B., Turgot, A., Wolf, S., and Tielbuerger, D. (2000a). "Acoustic normal mode fluctuation statistics in the 1995 SWARM internal wave scattering experiment," J. Acoust. Soc. Am. 107, 201-220.

Headrick, R. H., Lynch, J. F., Kemp, J. N., Newhall, A. E., von der Heydt, K., Apel, J., Badiey, M., Chiu, C.-S., Finette, S., Orr, M., Pasewark, B., Turgot, A., Wolf, S., and Tielbuerger, D. (2000b). "Modeling mode arrivals in the 1995 SWARM experiment acoustic transmissions," J. Acoust. Soc. Am. 107, 221-236.

Heaney, K. (2009). "A normal mode projection technique for array response synthesis in range-dependent environments," J. Acoust. Soc. Am. 126, 1036-1045.
Johnson, D. H., and Dudgeon, D. E. (1993). Array Signal Processing: Concepts and Techniques (PTR Prentice Hall, Upper Saddle River, NJ), Chap. 4.

Katsnelson, B. G., and S. A. Pereselkov (2000). "Low-frequency horizontal acoustic refraction caused by internal wave solitons in a shallow sea," Acoust. Phys. 46, 684-691.

Katsnelson, B. G., Grigorev, V., and Lynch, J. F. (2008). "Intensity fluctuations of midfrequency sound signals passing through moving nonlinear internal waves," J. Acoust. Soc. Am. 124, EL78-EL84.

Katsnelson, B. G., Grigorev, V., Badiey, M., and Lynch, J. F. (2009). "Temporal sound field fluctuations in the presence of internal solitary waves in shallow water," J. Acoust. Soc. Am. 126, EL41-EL48.

Lin, Y. T., Duda, T. F., and Lynch, J. F. (2009). "Acoustic mode radiation from the termination of a truncated nonlinear internal gravity wave duct in a shallow ocean area," J. Acoust. Soc. Am. 126, 1752-1765.

Lynch, J. F., Colosi, J. A., Gawarkiewicz, G., Duda, T. F., Pierce, A. D., Badiey, M., Katsnelson, B. G., Miller, J. H., Siegmann, W., Chiu, C.-S., and Newhall, A. (2006). "Consideration of fine-scale coastal oceanography and 3-D acoustics effects for the ESME sound exposure model," IEEE J. Oceanic Eng. 31, 33-48.

Lynch, J. F., Lin, Y.-T., Duda, T. F., and Newhall, A. E. (2010). "Acoustic ducting, reflection, refraction, and dispersion by curved nonlinear internal waves in shallow water," IEEE J. Oceanic Eng. 35, 12-27.

Meredith, R. W., and Nagle, S. M. (1999). "Coherence estimation for highfrequency narrowband $\mathrm{cw}$ pulsed signals in shallow water," J. Acoust. Soc. Am. 106, 828-836.

Mignerey, P. C., and Orr, M. H. (2004). "Observations of matched-field autocorrelation time in the South China Sea," IEEE J. Ocean. Eng. 29, $1280-1291$.

Newhall, A. E., Duda, T. F., von der Heydt, K., Irish, J. D., Kemp, J. N., Lerner, S. A., Liberatore, S. P., Lin, Y.-T., Lynch, J. F., Maffei, A. R., Morozov, A. K., Shmelev, A., Sellers, C. J., and Witzell, W. E. (2007). "Acoustic and oceanographic observations and configuration information for the WHOI moorings from the SW06 experiment," Woods Hole Oceanographic Institution Technical Report No. WHOI-2007-04, Woods Hole, MA.

Orr, M. H., Pasewark, B. H., Wolf, S. N., Lynch, J. F., Schroeder, T., and Chiu, C.-S. (2004). "South China Sea internal tide/internal waves-Impact on the temporal variability of horizontal array gain at $276 \mathrm{~Hz}$," IEEE J. Oceanic Eng. 29, 1292-1307.

Porter, M., (2011). "Normal mode models," Heat, Light and Sound Research, Inc., La Jolla, CA, http://oalib.hlsresearch.com/Modes/ (Last viewed November 2011).

Preisig, J. C., and Duda, T. F. (1997). "Coupled acoustic mode propagation through continental-shelf internal solitary waves," IEEE J. Oceanic Eng. 22, 256-269.

Rouseff, D., Turgut, A., Wolf, S. N., Finette, S., Orr, M. H., Pasewark, B. H., Apel, J. R., Badiey, M., Chiu, C.-S., Headrick, R. H., Lynch, J. F., Kemp, J. N., Newhall, A. E., von der Heydt, K., and Tielbuerger, D. (2002). "Coherence of acoustic modes propagating through shallow water internal waves," J. Acoust. Soc. Am. 111, 1655-1666.

Shroyer, E. L., Moum, J. N., and Nash, J. D. (2010). "Energy transformations and dissipation of nonlinear internal waves over New Jersey's continental shelf," Nonlin. Processes Geophys. 17, 345-360.

Tang, D., Moum, J. N., Lynch, J. F., Abbot, P., Chapman, R., Dahl, P. H., Duda, T. F., Gawarkiewicz, G., Glenn, S., Goff, J. A., Graber, H., Kemp, J., Maffei, A., Nash, J. D., and Newhall, A. (2007). "Shallow Water '06: A Joint Acoustic Propagation/Nonlinear Internal Wave Physics Experiment," Oceanography 20, 156-167.

Tielbuerger, D., Finette, S., and Wolf, S. (1997). "Acoustic propagation through the internal wave field in a shallow water waveguide," J. Acoust. Soc. Am. 101, 789-808.

Zhou, J., Zhang, X., and Rogers, P. H. (1991). "Resonant interaction of sound wave with internal solitons in the coastal zone," J. Acoust. Soc. Am. 90, 2042-2054. 